\title{
Estimating the decomposition of predictive information in multivariate systems
}

\author{
Luca Faes, ${ }^{1, *}$ Dimitris Kugiumtzis, ${ }^{2}$ Giandomenico Nollo, ${ }^{1}$ Fabrice Jurysta, ${ }^{3}$ and Daniele Marinazzo ${ }^{4}$ \\ ${ }^{1}$ BIOtech, Department of Industrial Engineering, University of Trento and IRCS Program, PAT-FBK, 38122 Trento, Italy \\ ${ }^{2}$ Department of Electrical and Computer Engineering, Aristotle University of Thessaloniki, Thessaloniki 54124, Greece \\ ${ }^{3}$ Sleep Laboratory, Department of Psychiatry, Université Libre de Bruxelles, Erasme Academic Hospital, 1050 Brussels, Belgium \\ ${ }^{4}$ Department of Data Analysis, University of Ghent, 9000 Ghent, Belgium
}

(Received 18 September 2014; published 6 March 2015)

\begin{abstract}
In the study of complex systems from observed multivariate time series, insight into the evolution of one system may be under investigation, which can be explained by the information storage of the system and the information transfer from other interacting systems. We present a framework for the model-free estimation of information storage and information transfer computed as the terms composing the predictive information about the target of a multivariate dynamical process. The approach tackles the curse of dimensionality employing a nonuniform embedding scheme that selects progressively, among the past components of the multivariate process, only those that contribute most, in terms of conditional mutual information, to the present target process. Moreover, it computes all information-theoretic quantities using a nearest-neighbor technique designed to compensate the bias due to the different dimensionality of individual entropy terms. The resulting estimators of prediction entropy, storage entropy, transfer entropy, and partial transfer entropy are tested on simulations of coupled linear stochastic and nonlinear deterministic dynamic processes, demonstrating the superiority of the proposed approach over the traditional estimators based on uniform embedding. The framework is then applied to multivariate physiologic time series, resulting in physiologically well-interpretable information decompositions of cardiovascular and cardiorespiratory interactions during head-up tilt and of joint brain-heart dynamics during sleep.
\end{abstract}

DOI: 10.1103/PhysRevE.91.032904

PACS number(s): 05.45.Tp, 87.19.1o, 02.50.Sk, 89.75.-k

\section{INTRODUCTION}

A topical subject in many domains of science and engineering is to investigate how the behavior of a complex system arises from the dynamical interactions among its component parts. This subject is commonly explored for an assigned target system, describing its dynamics in terms of the underlying sources of statistical dependence, identified either within the system itself or from its interactions with the other connected systems. A comprehensive approach to perform such a description is framed in the emerging field of information dynamics [1]. This approach has seen a surge of interest with the introduction of operational definitions of information storage [2] and information transfer [3] and with the subsequent development of efficient algorithms for the practical estimation of these measures [4-9]. Information storage, reflecting the information contained in the past of a dynamic process that is useful to predict its future, is closely (and inversely) related to a set of measures of entropy rate used in several contexts to assess the complexity of the process [10-12]; direct measures of information storage have been successfully used to study information processes in neuroscience [5], physiology [13] and artificial systems [2]. Information transfer is assessed by means of the very popular transfer entropy (TE) measure [3], which quantifies the directional effects between two processes as the information provided by the past of the driver about the future of the target, conditioned on the information already provided by

\footnotetext{
*Author to whom correspondence should be addressed; Laboratorio di Biosegnali, BIOtech, Università di Trento, via delle Regole 101, 38060 Mattarello, Trento, Italy; TEL: +39 0461 282773; FAX: +39 0461 883091; luca.faes@unitn.it
}

the past of the target. Also, given that the TE implements in the information-theoretic framework the ubiquitous concept of Granger causality [14], it has been proposed to assess information transfer between coupled systems in a wide variety of contexts [15-22].

The measures of information storage and information transfer reveal the sources of statistical dependence, contained, respectively, in the past states of the target system and in the past states of the driving systems, which contribute to the predictability of the future state of the target. Although these two measures are usually studied in isolation, they are not fully independent of each other. Indeed, they result as the components of a specific decomposition of the so-called predictive information about the assigned target process, which is a measure of the overall dependence between parts of a multivariate dynamical system [23]. In particular, variations in one of the components of the decomposition, induced, for instance, by a system transition, can be better understood by examining the corresponding variations in the other components. It is known that the information shared between subsystems is limited by the entropy rate [24,25], but can be biased by the complexity of the internal dynamics of the subsystems [26]; in fact, the TE may reflect changes in the internal properties of the subsystems and not only in the interactions among them [23]. Moreover, the measure of storage may reflect internal physical mechanisms in the target system but also storage mechanisms in another system that drives the observed one [5]. Following this line of reasoning, in the present study we show in simulations that it is strongly advisable to consider the information storage and transfer not as standalone measures of interdependence, but rather as parts of a whole framework for the analysis of dynamical dependences, where they are seen as components of the decomposition of predictive information. Moreover, we provide a complete 
framework for the joint estimation of the measures of information storage and information transfer, computed as factors in the decomposition of the predictive information about the target system (or subsystem) of a multivariate dynamical system. The estimation problem is far from trivial, as the computation of these quantities from experimental time series is hampered by both theoretical and practical questions. A major issue is the so-called curse of dimensionality [9], consisting in the fact that the reliability of information-theoretic estimates unavoidably degrades, increasing the dimension of the state spaces that need to be explored. In the computation of the predictive information and of its constituent terms, the dimension is kept high by the need of covering reasonably well the past history of the observed processes. This seriously hampers the estimation in the presence of multiple interacting systems and/or short-time series. Moreover, as with all information-theoretic functionals, any estimate of predictive information, information storage, and information transfer would show a bias dependent on the method used and on the characteristic of the data [27]. It turns out that the most common approaches to the estimation of information dynamics measures, e.g., those based on the uniform embedding of the observed multiple time series and exploiting classical entropy estimators such as those based on binning, yield highly biased estimates already for a small number of time series and low dimensions, which prevent any meaningful interpretation of the measures. To counteract all these issues, we propose here an approach that combines a procedure for dimensionality reduction based on nonuniform multivariate embedding [28] with the nearest-neighbor estimation of information-theoretic quantities [29]. While nonuniform embedding and nearest-neighbor estimation were previously proposed for the computation of causality measures [4,30], here they are employed with the broader perspective of quantifying jointly all the measures of information dynamics that contribute to the predictive information of a target system embedded in a network.

\section{PREDICTIVE INFORMATION DECOMPOSITION}

We start by presenting an information-theoretic framework that provides a set of measures of information dynamics designed to characterize the temporal statistical structure of interacting dynamical systems [1,23]. Considering an overall multivariate system composed of several potentially connected subsystems, in this study we focus attention on an assigned target system (or subsystem) and analyze the amount of information carried by its present state that can be predicted from the knowledge of the past of the whole multivariate system. This information is known as predictive information and can be intuitively understood as a measure of how the uncertainty about the evolution of the target system can be reduced by learning the past of the whole network of interacting systems [23].

Given a set of $M$ interacting dynamical systems, let us assume that the course of visitation of the system states can be described as a multivariate stationary stochastic process. We consider the problem of estimating the factors in the decomposition of the predictive information relevant to the target process $Y$, considering $X$ as the source process and grouping the remaining $M-2$ processes into the vector $\mathbf{Z}=\left[Z^{(1)} \cdots Z^{(M-2)}\right]$.
Let us further denote by $X_{n}, Y_{n}$, and $\mathbf{Z}_{n}$ the random variables obtained by sampling the processes at the present time $n$ and by $X_{n}^{-}=\left[X_{n-1} X_{n-2} \cdots\right], Y_{n}^{-}=\left[Y_{n-1} Y_{n-2} \cdots\right]$, and $\mathbf{Z}_{\mathbf{n}}^{-}=\left[\mathbf{Z}_{\mathbf{n}-\mathbf{1}} \oplus \mathbf{Z}_{\mathbf{n}-\mathbf{2}} \oplus \cdots\right]$ the vector variables describing the past of the processes ( $\oplus$ denotes vector concatenation). Then the prediction entropy (PE) of the target process $Y$ measures the predictive information defined as the amount of information carried by the present of $Y$ that can be predicted by the whole past of the overall process $\{X, Y, \mathbf{Z}\}[23]$ :

$$
\begin{aligned}
P_{Y} & =I\left(Y_{n} ; X_{n}^{-} \oplus Y_{n}^{-} \oplus \mathbf{Z}_{n}^{-}\right) \\
& =H\left(Y_{n}\right)-H\left(Y_{n} \mid X_{n}^{-} \oplus Y_{n}^{-} \oplus \mathbf{Z}_{n}^{-}\right),
\end{aligned}
$$

where $I(\cdot)$ stands for mutual information (MI) and $H(\cdot)$ and $H(\cdot \mid \cdot)$ denote, respectively, entropy and conditional entropy. In order to put in evidence the statistical dependences related to the information actively stored in the target process and transferred to it from the source process and from the other processes, the PE can be conveniently decomposed by exploiting the chain rule for mutual information decomposition [23,31] as

$$
P_{Y}=S_{Y}+T_{\mathbf{Z} \rightarrow Y}+T_{X \rightarrow Y \mid \mathbf{Z}}
$$

where

$$
S_{Y}=I\left(Y_{n} ; Y_{n}^{-}\right)=H\left(Y_{n}\right)-H\left(Y_{n} \mid Y_{n}^{-}\right)
$$

measures the information storage relevant to the target process [2] and denotes here the storage entropy (SE), while

$$
\begin{aligned}
T_{\mathbf{Z}_{\rightarrow Y}}= & I\left(Y_{n} ; \mathbf{Z}_{n}^{-} \mid Y_{n}^{-}\right)=H\left(Y_{n} \mid Y_{n}^{-}\right)-H\left(Y_{n} \mid Y_{n}^{-} \oplus \mathbf{Z}_{n}^{-}\right), \\
T_{X \rightarrow Y \mid \mathbf{Z}} & =I\left(Y_{n} ; X_{n}^{-} \mid Y_{n}^{-} \oplus \mathbf{Z}_{n}^{-}\right) \\
& =H\left(Y_{n} \mid Y_{n}^{-} \oplus \mathbf{Z}_{n}^{-}\right)-H\left(Y_{n} \mid X_{n}^{-} \oplus Y_{n}^{-} \oplus \mathbf{Z}_{n}^{-}\right)
\end{aligned}
$$

are well-known measures of information transfer quantifying, respectively, the TE from $\mathbf{Z}$ to $Y$ [3] and the partial transfer entropy (PTE) from $X$ to $Y$ conditioned to $\mathbf{Z}[7,8,32,33]$.

The framework presented above is developed under the assumption that the joint process $\{X, Y, \mathbf{Z}\}$ is stationary and ergodic, which means that the probability density functions of any variable derived from the process do not change over time. This allows us to drop the dependence on the time index $n$ for the information dynamics measures defined in (1)-(5) and to provide their estimation based on a single process realization by pooling data over time. A nonstationary definition of the framework may be achieved intuitively, e.g., according to the formulations proposed in [23].

In order to understand the theoretical properties of the measures of information dynamics presented above, we performed their evaluation on an analytically tractable model of linearly interacting Gaussian systems. This class of systems allows us to compute exact theoretical values of predictive information, information storage, and information transfer rather than their statistical estimates, thereby isolating the fundamental properties of each measure from the unavoidable bias that is carried by any estimation approach. The exact computation relies on the correspondence between the conditional entropy and the prediction error variance of linear regression models [34], which was exploited to provide an exact expression for the TE based on the vector autoregressive (VAR) representation 

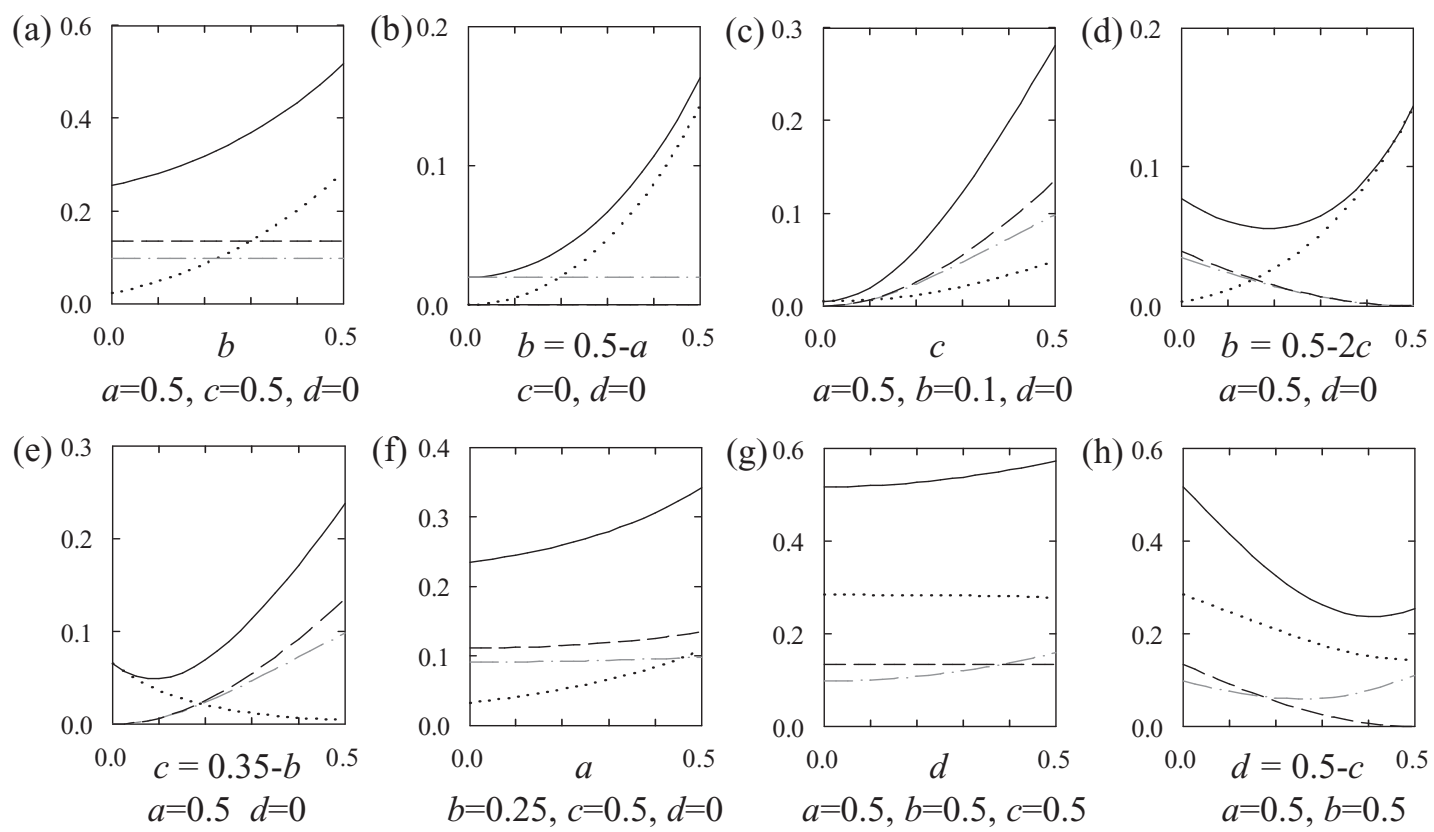

FIG. 1. Exact computation of the measures of information dynamics for the benchmark simulation example of Eq. (6). Plots depict the trends of PE (solid lines), SE (dotted lines), TE (dashed lines), and PTE (gray dash-dotted lines) computed with varying one or two of the simulation parameters (as indicated in the $x$-axis label of each plot), keeping the remaining parameters at the constant values specified below the plot.

of multivariate Gaussian processes [35,36]. In this study we generalize the approach to the exact computation of the measures of information dynamics (the formal derivation is detailed in the Appendix) so as to allow a highly reliable comparative evaluation of the properties of these measures. The considered benchmark system is defined by the equations

$$
\begin{aligned}
& X_{n}=-a X_{n-2}+Z_{n-1}+U_{n}^{X}, \\
& Y_{n}=-b Y_{n-2}+c X_{n-1}+d Z_{n-1}+U_{n}^{Y}, \\
& Z_{n}=U_{n}^{Z},
\end{aligned}
$$

where $U_{n}^{X}, U_{n}^{Y}$, and $U_{n}^{Z}$ are uncorrelated Gaussian white noise processes with zero mean and unitary variance. The causal statistical structure of the processes in (6) is such that the target $Y$ has autonomous dynamics determined by the parameter $b$ and is affected by the process $X$ (directly through the link weighted by the parameter $c$ ) and by the process $Z$ (both directly through the link weighted by $d$ and indirectly through the connection $Z \rightarrow X \rightarrow Y$ ); the driver process $X$ also shows internal dynamics weighted by $a$ and receives direct causal influences from $Z$, which is an autonomous random process without temporal dynamics.

Figure 1 reports the trends of the measures of information dynamics obtained for representative combinations of the simulation parameters. The figure shows how, depending on the strength of the underlying causal connections, the predictive information of the target $Y$ is decomposed in different amounts of information stored in $Y$ and transferred to $Y$ from $X$ and $Z$. The information storage assessed by the SE increases with the strength of the autodependence effects in $Y$ modulated by the parameter $b$ [Figs. 1(a) and 1(b)], but increases also with the strength of the causal effects from $X$ to $Y$ modulated by $c$ [Fig. 1(c)]. Similar reciprocal changes in the strength of autodependence and causal effects may produce either an increase or a decrease of the SE, as shown, respectively, in Figs. 1(d) and 1(e). Moreover, the SE of $Y$ is sensitive also to the intrinsic dynamics of $X$ [Fig. 1(f)]. These results indicate that the information storage quantifies the overall dependence of the present of the target process on its past as the result of multiple causation mechanisms, which incorporate the internal dynamics of the target process, but also dynamical properties of the source process and the causal interactions from source to target. This is in line with the notion that the task of actively storing information in the past of a target process is subserved both by mechanisms of internal memory in the target and by mechanisms of input-driven storage determined by causally connected source processes [5,37]. As to the information transfer, we see that both TE and PTE are zero in the absence of causal connections (from $Z$ to $Y$ and from $X$ to $Y$, respectively), do not depend on the strength of the internal dynamics of $Y$ modulated by the parameter $b$ [Figs. 1(a) and 1(b)], and increase with the strength of the causal interactions from $X$ to $Y$ modulated by $c$ [Figs. 1(c)-1(e)]. The distinction between TE and PTE stands in the fact that the first measures the transfer of information along both the direct causal connection $Z \rightarrow Y$ and indirect connection $Z \rightarrow X \rightarrow Y$, while the second measures only the direct transfer along the connection $X \rightarrow Y$ and is not sensitive to the interaction between $Z$ and $Y$ [Figs. 1(g) and 1(h)]. These results pinpoint the usefulness of the information transfer as a measure reflecting the causal interactions in the observed multivariate system. Nevertheless, besides reflecting the causal interactions, the information transfer may be sensitive also to the complexity of the internal dynamics of the driver system, as shown in Fig. 1(f), where TE and PTE vary (slightly in this example) with the parameter $a$. In general, the trends 
observed in Fig. 1 for the measures of information storage and transfer indicate the importance of evaluating the complexity of the individual systems, and not only their causal interaction, to properly assess how the target dynamics arise from the causal structure of the overall system. Moreover, since the contributions of the various terms composing the predictive information may be different depending on the underlying causal structure, it is important to evaluate all measures in a unified framework rather than in isolation. This confirms previous observations remarking that functionally relevant changes in the dynamics of the coupled systems can be more properly described through a combined evaluation of the decomposition terms rather than through an analysis based on single measures of storage and transfer $[5,23,38]$.

\section{ESTIMATION APPROACH}

All measures of information dynamics defined in Sec. II can be derived from computations of the entropy of the present variable of the target process $Y_{n}$ conditioned to the semi-infinite past of the observed processes $X_{n}^{-}, Y_{n}^{-}$, or $\mathbf{Z}_{n}^{-}$. Such computations are obviously a daunting task in practical time-series analysis because they require entropy estimation for high-dimensional vector variables even when the past history of the observed processes is truncated to some small lag. To tackle the estimation issue, we here propose a two-step procedure based first on performing a parsimonious state space reconstruction that selects from each process the past (lagged) variables that are more informative to $Y_{n}$ [28] and then exploits only the selected variables to estimate predictive information, information storage, and information transfer. In both steps, estimations are performed using a nearest-neighbor approach that adopts an efficient strategy to reduce the bias arising from the computation of entropies involving variables of different dimension [29].

\section{A. Embedding schemes}

The first crucial step in the estimation of information dynamics is to provide a reasonable approximation of the infinite-dimensional past states of the observed systems. In the analysis of dynamical systems, this is achieved through the state space reconstruction of the observed multivariate process. When the aim of the analysis is the computation of information dynamics, state space reconstruction serves, rather than to infer the properties of the attractor of the underlying dynamical system, to identify the most relevant finite set of state variables to be taken as representative of the system dynamics. To this end, reconstruction is viewed as a way to sample the past of the processes $X_{n}^{-}, Y_{n}^{-}$, and $\mathbf{Z}_{n}^{-}$in order to provide as much information as possible about the dynamics of the target variable $Y_{n}$. Two possible approaches are described in the following.

\section{Uniform embedding}

The most obvious approach to state space reconstruction is to follow a uniform embedding scheme [39] whereby the past of each considered process is approximated using a predetermined number of variables equally spaced in time.
In the univariate case, only the state space of a scalar process needs to be reconstructed, e.g., for the computation of the SE in (3). In this case the uniform embedding vector descriptive of $Y_{n}^{-}$is $V_{n}^{Y}=\left[Y_{n-m_{Y}} Y_{n-2 m_{Y}} \cdots Y_{n-d_{Y} m_{Y}}\right]$, where $d_{Y}$ and $m_{Y}$ are the embedding dimension and delay time, respectively. The computation of the TE in (4) requires a mixed embedding approximating the past of the joint process $\{Y, \mathbf{Z}\}$; in this case, the uniform embedding vector approximating $Y_{n}^{-} \oplus \mathbf{Z}_{n}^{-}$is $V_{n}^{Y \mathbf{Z}}=V_{n}^{Y} \oplus\left[\mathbf{Z}_{n-m_{Z}} \mathbf{Z}_{n-2 m_{Z}} \cdots \mathbf{Z}_{n-d_{Z} m_{Z}}\right]$. In a similar way, a full mixed embedding representing the past of the whole joint process $\{X, Y, Z\}$ is required to compute either the $\mathrm{PE}$ in (1) or the PTE in (5); in this case, the whole past $\mathbf{Z}_{n}^{-}$ is approximated by the uniform embedding vector $V_{n}{ }^{X Y \mathbf{Z}}=$ $V_{n}^{Y \mathbf{Z}} \oplus\left[X_{n-m_{X}} X_{n-2 m_{X}} \cdots X_{n-d_{X} m_{X}}\right]$.

\section{Nonuniform embedding}

As an alternative to uniform embedding, we propose a nonuniform embedding approach, which follows the philosophy of some previous work from our group [28,30,40,41], seeking maximum relevance, and at the same time minimum redundancy, in the selection of components to be included in the embedding vector. The approach is based on the progressive selection, from a set of candidate components $\Omega$ including the lagged variables that sample the past of the relevant processes up to a maximum lag $L$, of the variables that are the most informative about the target variable $Y_{n}$. In the case of the univariate state space reconstruction of the process $Y$, the initial set of candidate components will be $\Omega^{Y}=$ $\left\{Y_{n-1}, \ldots, Y_{n-L}\right\}$ and the selection procedure will approximate the past $Y_{n}^{-}$with a nonuniform embedding vector $V_{n}^{Y}=Y_{n}^{Y}$ composed of the $d_{Y}$ most relevant lagged variables of $Y$. In the case of the mixed embedding of $\{Y, \mathbf{Z}\}$, the candidate set will be $\Omega^{Y \mathbf{Z}}=\Omega^{Y} \cup\left\{\mathbf{Z}_{n-1}, \ldots, \mathbf{Z}_{n-L}\right\}$ and the selection procedure will approximate $Y_{n}^{-} \oplus \mathbf{Z}_{n}^{-}$with the embedding vector $V_{n}^{Y \mathbf{Z}}=Y_{n}^{Y \mathbf{Z}} \oplus \mathbf{Z}_{n}^{Y \mathbf{Z}}$, where $Y_{n}^{Y \mathbf{Z}}$ and $\mathbf{Z}_{n}^{Y \mathbf{Z}}$ denote the selected components belonging, respectively, to $Y$ and $\mathbf{Z}$. With similar notation, for the full mixed embedding of $\{X, Y, \mathbf{Z}\}$ the initial candidate set will be $\Omega^{X Y \mathbf{Z}}=\Omega^{Y \mathbf{Z}} \cup\left\{X_{n-1}, \ldots, X_{n-L}\right\}$ and the nonuniform embedding vector will be $V_{n}^{X Y \mathbf{Z}}=X_{n}^{X Y \mathbf{Z}} \oplus$ $Y_{n}^{X Y \mathbf{Z}} \oplus \mathbf{Z}_{n}^{X Y \mathbf{Z}}$. With respect to uniform embedding where the embedding vectors contain a predefined number of variables for each process, the vectors resulting from nonuniform embedding contain only the variables found to be relevant to the description of $Y_{n}$. This parsimonious selection of variables favors entropy estimation as it reduces the dimension of the reconstructed state space. In the following we describe the selection procedure considering the generic candidate set $\Omega$ and the approach adopted to estimate information-theoretic quantities.

The procedure starts with an empty embedding vector $V_{n}^{0}=$ [.] and, at the step $d \geqslant 1$, given the embedding vector formed at the previous step $V_{n}^{d-1}$, each candidate component $W_{n} \in \Omega \backslash$ $V_{n}^{d-1}(\backslash$ denotes subtraction from a set) is tested computing the conditional MI (CMI) $I\left(Y_{n}, W_{n} \mid V_{n}^{d-1}\right)$ and the component selected is that yielding the maximum $\mathrm{CMI}$ :

$$
\hat{W}_{n}=\arg \max _{W_{n}} I\left(Y_{n}, W_{n} \mid V_{n}^{d-1}\right) .
$$

After selection, the component $\hat{W}_{n}$ is tested for significance using an empirical approach based on surrogate data [30]. 
Specifically, the CMI $I\left(Y_{n}, \hat{W}_{n} \mid V_{n}^{d-1}\right)$ is compared with a threshold taken as the $100(1-\alpha)$ th percentile of its empirical null distribution estimated over $N_{r}$ random counterparts obtained by shuffling randomly and independently the points of $\hat{W}_{n}$ and the points of $Y_{n}$. Then if the original CMI is above the threshold, the component $\hat{W}_{n}$ is included in the embedding vector to form $V_{n}^{d}=\hat{W}_{n} \oplus V_{n}^{d-1}$; otherwise it is discarded and the procedure terminates with an embedding vector $V_{n}=V_{n}^{d-1}$.

In this study, each CMI is estimated using the $k$-nearestneighbor approach, based on estimating entropy sums through a neighbor search in the space of higher dimension and range searches in the projected subspaces of lower dimensions [29]. Specifically, to estimate the CMI $I\left(Y_{n}, W_{n} \mid V_{n}^{d-1}\right)$ we first express it as the sum of four entropies

$$
\begin{aligned}
& I\left(Y_{n}, W_{n} \mid V_{n}^{d-1}\right) \\
&= H\left(Y_{n} \oplus V_{n}^{d-1}\right)-H\left(V_{n}^{d-1}\right) \\
&-H\left(Y_{n} \oplus W_{n} \oplus V_{n}^{d-1}\right)+H\left(W_{n} \oplus V_{n}^{d-1}\right) .
\end{aligned}
$$

The entropy in the $(d+1)$-dimensional space is estimated, using the maximum norm to calculate distances, as

$$
\hat{H}\left(Y_{n} \oplus W_{n} \oplus V_{n}^{d-1}\right)=-\psi(k)+\psi(N)+(d+1)\left\langle\ln \varepsilon_{n}\right\rangle,
$$

where $\psi$ is the digamma function, $N$ is the total number of available $(d+1)$-dimensional points $Y_{n} \oplus W_{n} \oplus V_{n}^{d-1}, \varepsilon_{n}$ is twice the distance from $Y_{n} \oplus W_{n} \oplus V_{n}^{d-1}$ to its $k$ th neighbor, and $\langle\cdot\rangle$ denotes average over all $n$. As we use the natural logarithm in (9), the entropy is measured in natural units (nats). Then the three other entropy terms of (8) are estimated by treating the relevant variables as projections from the $(d+1)$ dimensional space, yielding

$$
\begin{aligned}
& \hat{H}\left(W_{n} \oplus V_{n}^{d-1}\right)=\psi(N)-\left\langle\psi\left(N_{W_{n} V_{n}^{d-1}}+1\right)\right\rangle+d\left\langle\ln \varepsilon_{n}\right\rangle, \\
& \hat{H}\left(Y_{n} \oplus V_{n}^{d-1}\right)=\psi(N)-\left\langle\psi\left(N_{Y_{n} V_{n}^{d-1}}+1\right)\right\rangle+d\left\langle\ln \varepsilon_{n}\right\rangle, \\
& \hat{H}\left(V_{n}^{d-1}\right)=\psi(N)-\left\langle\psi\left(N_{V_{n}^{d-1}}+1\right)\right\rangle+(d-1)\left\langle\ln \varepsilon_{n}\right\rangle,
\end{aligned}
$$

where $N_{W_{n} V_{n}^{d-1}}, N_{Y_{n} V_{n}^{d-1}}$, and $N_{V_{n}^{d-1}}$ are the number of points whose distance from $W_{n} \oplus V_{n}^{d-1}, Y_{n} \oplus V_{n}^{d-1}$, and $V_{n}^{d-1}$, respectively, is strictly less than $\varepsilon_{n} / 2$. Then the CMI estimate results simply by substituting (9) and (10) into (8):

$$
\begin{aligned}
\hat{I}\left(Y_{n}, W_{n} \mid V_{n}^{d-1}\right)= & \psi(k)+\left\langle\psi\left(N_{V_{n}^{d-1}}+1\right)-\psi\left(N_{W_{n} V_{n}^{d-1}}+1\right)\right. \\
& \left.-\psi\left(N_{Y_{n} V_{n}^{d-1}}+1\right)\right\rangle .
\end{aligned}
$$

\section{B. Estimation of information dynamics measures}

In this section we describe how the measures of information dynamics defined in Eqs. (1)-(5) were estimated by means of the $k$-nearest-neighbor approach using the embedding vectors determined by the uniform or nonuniform embedding schemes presented in Sec. III A.

\section{Estimation of information storage}

To estimate the SE relevant to the target process $Y$, univariate (uniform or nonuniform) embedding is first performed for the single process $Y$ to get the $d_{Y}$-dimensional embedding vector $V_{n}^{Y}=Y_{n}^{Y}$ composed of lagged variables from $Y$. Then, expressing the SE as $S_{Y}=H\left(Y_{n}\right)-H\left(Y_{n} \oplus V_{n}^{Y}\right)+H\left(V_{n}^{Y}\right)$, the term $H\left(Y_{n} \oplus V_{n}^{Y}\right)$ is estimated through a neighbor search in the $\left(d_{Y}+1\right)$-dimensional space, while the terms $H\left(Y_{n}\right)$ and $H\left(V_{n}^{Y}\right)$ are estimated through range searches in the spaces of dimension 1 and $d_{Y}$. This results in the estimate

$$
\hat{S}_{Y}=\psi(N)+\psi(k)-\left\langle\psi\left(N_{Y_{n}}+1\right)+\psi\left(N_{V_{n}^{Y}}+1\right)\right\rangle,
$$

where $N_{Y_{n}}$ and $N_{V_{n}^{Y}}$ are the number of points whose distance from $Y_{n}$ and $V_{n}^{Y}$, respectively, is strictly less than the distance from $Y_{n} \oplus V_{n}^{Y}$ to its $k$ th neighbor.

\section{Estimation of information transfer}

The estimation of the TE and PTE measures defined in (4) and (5) exploits mixed embedding schemes aimed at describing the past of multivariate systems. In particular, to estimate the TE from $\mathbf{Z}$ to $Y$, mixed (uniform or nonuniform) embedding is performed for the joint process $\{Y, \mathbf{Z}\}$ to get the $\left(d_{Y}+d_{Z}\right)$-dimensional embedding vector $V_{n}^{Y Z}=Y_{n}^{Y \mathbf{Z}} \oplus$ $\mathbf{Z}_{n}^{Y \mathbf{Z}}$. Then expressing the TE as $T_{\mathbf{Z} \rightarrow Y}=H\left(Y_{n} \oplus Y_{n}^{Y \mathbf{Z}}\right)-$ $H\left(Y_{n}^{Y \mathbf{Z}}\right)-H\left(Y_{n} \oplus V_{n}^{Y \mathbf{Z}}\right)+H\left(V_{n}^{Y \mathbf{Z}}\right)$, the term $H\left(Y_{n} \oplus V_{n}^{Y \mathbf{Z}}\right)$ is estimated through a neighbor search in the $\left(d_{Y}+d_{Z}+1\right)$ dimensional space, while the three other terms are estimated through range searches in the spaces of dimension $d_{Y}+1$, $d_{Y}+d_{Z}$, and $d_{Y}$. This results in the estimate

$$
\begin{aligned}
\hat{T}_{Z \rightarrow Y}= & \psi(k)+\left\langle\psi\left(N_{Y_{n}^{Y Z}}+1\right)-\psi\left(N_{Y_{n} Y n}+1\right)\right. \\
& \left.-\psi\left(N_{V_{n}^{Y Z}}+1\right)\right\rangle
\end{aligned}
$$

where $N_{Y_{n}^{Y Z}}, N_{Y_{n} Y_{n}^{Y Z}}$, and $N_{V_{n}^{Y Z}}$ are the number of points whose distance from $Y_{n}^{Y \mathbf{Z}}, Y_{n} \oplus Y_{n}^{Y \mathbf{Z}}$ and $V_{n}^{Y \mathbf{Z}}$, respectively, is strictly less than the distance from $Y_{n} \oplus V_{n}^{Y Z}$ to its $k$ th neighbor.

To estimate the PTE from $X$ to $Y$ conditioned to $\mathbf{Z}$, mixed embedding is performed for the joint process $\{X, Y, \mathbf{Z}\}$ to get the $\left(d_{X}+d_{Y}+d_{Z}\right)$-dimensional embedding vector $V_{n}^{X Y \mathbf{Z}}=X_{n}^{X Y \mathbf{Z}} \oplus Y_{n}^{X Y \mathbf{Z}} \oplus \mathbf{Z}_{n}^{X Y \mathbf{Z}}$. Then expressing the PTE

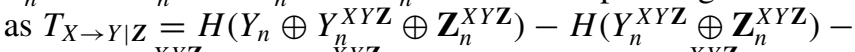
$H\left(Y_{n} \oplus V_{n}^{X Y \mathbf{Z}}\right)+H\left(V_{n}^{X}{ }^{X \mathbf{Z}}\right)$, the term $H\left(Y_{n} \oplus V_{n}^{X Y \mathbf{Z}}\right)$ is estimated through a neighbor search in the $\left(d_{X}+d_{Y}+d_{Z}+1\right)$ dimensional space, while the three other terms are estimated through range searches in the spaces of dimension $d_{Y}+d_{Z}+$ $1, d_{Y}+d_{Z}$, and $d_{X}+d_{Y}+d_{Z}$. This results in the estimate

$$
\begin{aligned}
\hat{T}_{X \rightarrow Y \mid Z}= & \psi(k)+\left\langle\psi\left(N_{Y_{n}^{X Y Z} Z_{n}^{X Y Z}}+1\right)\right. \\
& \left.-\psi\left(N_{Y_{n} Y_{n}^{X Y Z} Z_{n}^{X Y Z}}+1\right)-\psi\left(N_{V_{n}^{X Y Z}}+1\right)\right\rangle,
\end{aligned}
$$

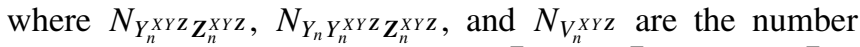
of points whose distance from $Y_{n}^{X Y \mathbf{Z}} \oplus \mathbf{Z}_{n}^{X Y \mathbf{Z}}, Y_{n} \oplus Y_{n}^{X Y \mathbf{Z}} \oplus$ $\mathbf{Z}_{n}^{X Y \mathbf{Z}}$, and $V_{n}^{X Y \mathbf{Z}}$, respectively, is strictly less than the distance from $Y_{n} \oplus V_{n}^{X Y Z}$ to its $k$ th neighbor.

\section{Estimation of predictive information and its decomposition based on full embedding}

Estimation of the PE about the target process $Y$ is performed following the same full mixed embedding performed for the whole joint process $\{X, Y, \mathbf{Z}\}$ to estimate the PTE. Specifically, the embedding vector $V_{n}^{X Y Z}$ is used to express the PE as $P_{Y}=$ $H\left(Y_{n}\right)-H\left(Y_{n} \oplus V_{n}^{X Y \mathbf{Z}}\right)+H\left(V_{n}^{X Y \mathbf{Z}}\right)$, so its nearest-neighbor 
estimate becomes

$$
\hat{P}_{Y}=\psi(k)+\psi(N)-\left\langle\psi\left(N_{Y_{n}}+1\right)+\psi\left(N_{V_{n}^{X Y Z}}+1\right)\right\rangle,
$$

where $N_{Y_{n}}$ and $N_{V_{n}^{X Y Z}}$ are the number of points whose distance from $Y_{n}$ and $V_{n}^{X Y Z}$ is strictly less than the distance from $Y_{n} \oplus V_{n}^{X Y Z}$ to its $k$ th neighbor. Note that, because of the different bias resulting from estimating the various measures of information dynamics, the equivalence between the PE and the sum of SE, TE, and PTE stated in (2) does not hold for the estimates resulting from (15) and from (12)-(14). In this study we investigate the difference between $\hat{P}_{Y}$ and $\hat{S}_{Y}+\hat{T}_{\mathbf{Z} \rightarrow Y}+\hat{T}_{X \rightarrow Y \mid \mathbf{Z}}$ from the point of view of comparing uniform embedding and nonuniform embedding schemes as to their ability in estimating the prediction entropy and the terms of its decomposition.

\section{SIMULATION STUDY}

In this section we test the proposed estimation approach on well-known linear stochastic (VAR models) and nonlinear chaotic (coupled Hénon maps) multivariate systems, comparing uniform and nonuniform embedding schemes regarding their ability to quantify the various measures of information dynamics under different coupling conditions. While the considered systems are rather simple, their utilization allows a clear understanding of the performance of the proposed estimators: In VAR processes the estimates of information dynamics can be compared with their theoretical values (see the Appendix), while Hénon systems have been exhaustively studied [30]. Moreover, simulation studies were conducted both in standard and in more challenging conditions, induced by increased interaction lags or a higher number of interacting processes.

In all simulations, 100 realizations of the simulated processes, each lasting 512 points, were generated for each of the selected values of the coupling parameter $C$. Uniform embedding was performed by setting for each simulation the optimal values of the embedding parameters from the system equations. Nonuniform embedding was performed by testing for the significance of each selected component by means of $N_{r}=100$ replicas generated by a random and independent shift of the target variable and the selected component and by setting $\alpha=0.05$ as the statistical significance level. The number of neighbors used for all entropy estimations was set to $k=10$. This choice was based on the results of previous explorations of a wide range of values of $k$ in the estimation of MI and CMI based on nearest neighbors [28,30]. In general, $k$-nearest-neighbor methods for the estimation of information measures were found to be stable and not significantly affected by the choice of $k$ [29]. All time series were normalized to zero mean and unit standard deviation before computation of the measures.

\section{A. Linear stochastic processes}

The first system considered is a linear stochastic VAR system described by the equations

$X_{n}=0.8485 X_{n-1}-0.36 X_{n-2}+U_{n}^{X}$,

$$
\begin{aligned}
Y_{n}= & C\left(X_{n-\delta}+Z_{n-\delta}\right)+\left(1-\frac{C}{2}\right)\left(0.8485 Y_{n-1}-0.36 Y_{n-2}\right) \\
& +U_{n}^{Y}, \\
Z_{n}= & 0.8485 Z_{n-1}-0.36 Z_{n-2}+U_{n}^{Z},
\end{aligned}
$$

where $U_{n}^{X}, U_{n}^{Y}$, and $U_{n}^{Z}$ are uncorrelated Gaussian white noise processes of unitary variance. The parameters in (16) were chosen to simulate a situation typical of cardiovascular and cardiorespiratory variability analysis, where a target process may exhibit autonomous oscillations and also be driven by other processes oscillating at the same frequency [41]. In this simulation we used two different settings for the delay of the coupling imposed contemporaneously from $X$ to $Y$ and from $Z$ to $Y: \delta=1$ and 10 . In both cases the coupling parameter $C$ was varied from 0 to 1 in steps of 0.1 .

In this simulation we compared the exact values of the measures of information dynamics, obtained under the assumption of Gaussianity using the formulation detailed in the Appendix, with the estimates resulting from the application of the approach presented in Sec. III. The comparison between exact and estimated trends allowed us to assess the bias of our estimation approach. The analysis was performed computing PE, SE, TE, and PTE as a function of the coupling parameter $C$, taking $Y$ as the target process and $X$ as the source process. Uniform embedding was performed with unitary embedding lags $\left(m_{X}=m_{Y}=m_{Z}=1\right)$ and optimal values for the embedding dimensions $\left(d_{X}=d_{Y}=d_{Z}=2\right.$ in the first simulation with $\delta=1$ and $d_{X}=d_{Y}=d_{Z}=10$ in the second simulation with $\delta=10$ ). Nonuniform embedding was performed including $L=10$ past components in the set of candidates for each considered process.

The results are reported in Fig. 2 for $\delta=1$ and in Fig. 3 for $\delta=10$. In general, we found that all estimates of information dynamics have a negative bias, i.e., tend to underestimate the actual quantities. This bias was relatively small and did not impair the detection of trends of the measures (e.g., for varying coupling parameter) when estimation was based on nonuniform embedding. In contrast, the estimation based on uniform embedding degraded as the coupling delay increased. Indeed, with $\delta=1$ (Fig. 2) the two schemes yielded similar results, with only a slightly better performance of nonuniform embedding for high values of $C$, noticeable at the lower bias of TE and especially PTE. With $\delta=10$ (Fig. 3), results were almost the same as with $\delta=1$ for nonuniform embedding, while the estimates based on uniform embedding were highly biased. In particular, the estimates based on uniform embedding were almost unresponsive to variations in the coupling parameter (e.g., the estimated PTE was very low and almost flat for all values of $C$ ), while those based on nonuniform embedding approximated well the theoretical profiles. The better performance of nonuniform embedding can be ascribed to its ability to pick up only the relevant terms for describing the dynamics of the target process, regardless of their lag. In contrast, uniform embedding covering long interaction delays has to include many components in the embedding vectors, thus hindering the estimation computed in the presence of short realizations of the investigated processes.

The comparison between the two possible ways of assessing the overall predictive information showed that the estimation 
(a)
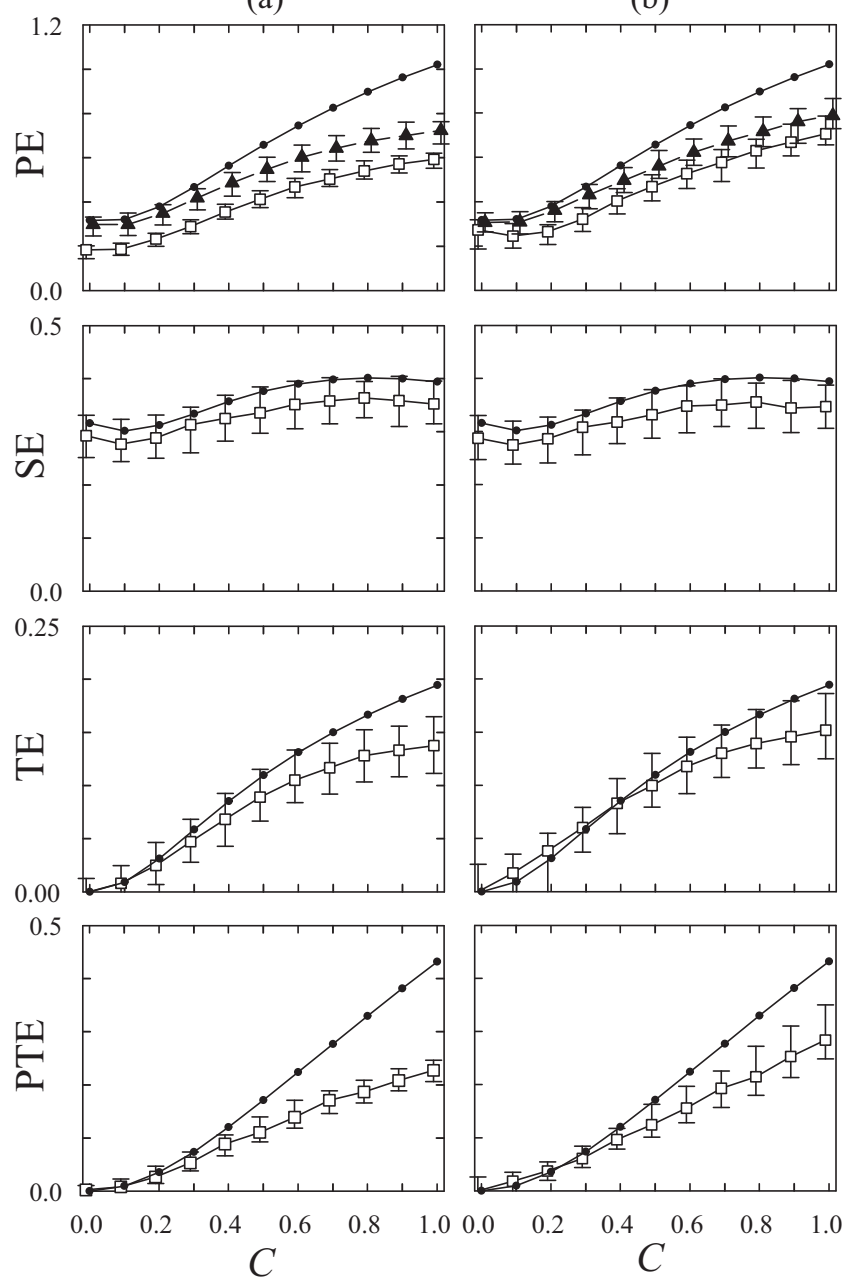

FIG. 2. Estimation of measures of information dynamics for simulation A (linear VAR process) with delay $\delta=1$. Plots depict the theoretical values (dots) and the estimated values (black and white symbols) (median and 10th-90th percentiles over 100 process realizations) of the PE of $Y$, SE of $Y$, TE from $Z$ to $Y$, and PTE from $X$ to $Y$ conditioned to $Z$, computed as a function of the coupling parameter $C$ by means of (a) the uniform embedding approach and (b) the nonuniform embedding approach. The Prediction Entropy is computed through the direct estimation (white squares) as well as the sum of the estimated SE, TE and PTE (black triangles).

bias is lower when PE is computed by summing up the storage and transfer estimates of Eqs. (12)-(14) than when it is directly computed in the full space of $\{X, Y, Z\}$ through Eq. (15). Thus, it seems that practical estimation of the predictive information may be performed more effectively by estimating separately each of the terms that contribute to the generation of predictable dynamics. If we would use the other approach (i.e., computing all storage and transfer terms with reference to the largest dimensional space) there would be no difference between the direct estimation of the PE and the sum of the estimated SE, TE, and PTE. So the difference is due to defining a different largest space for each of the three decomposed terms. A possible explanation for such a difference may be the fact that the bias of each individual term is small since the corresponding CMI is given as the difference between two (a)
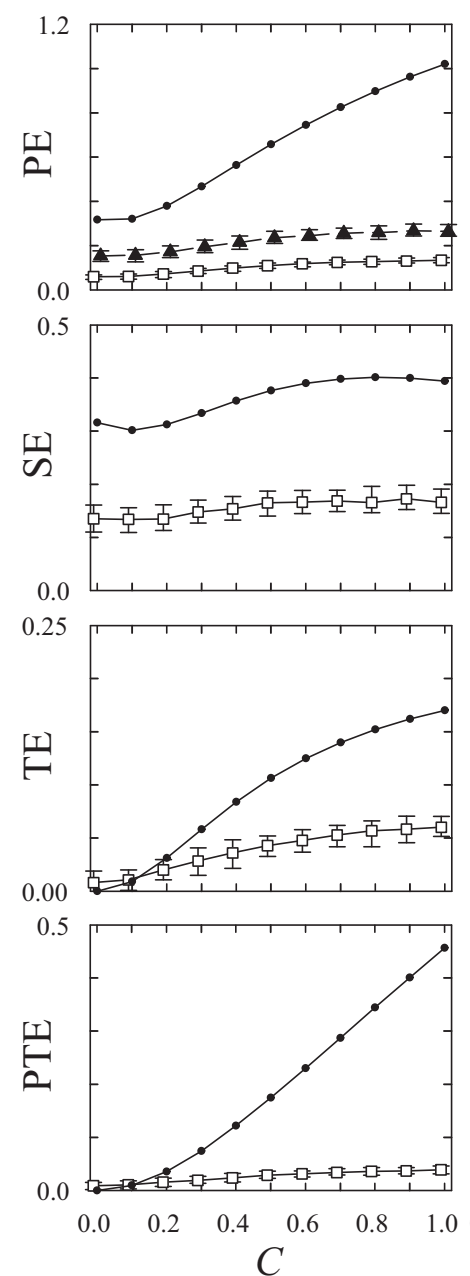

(b)
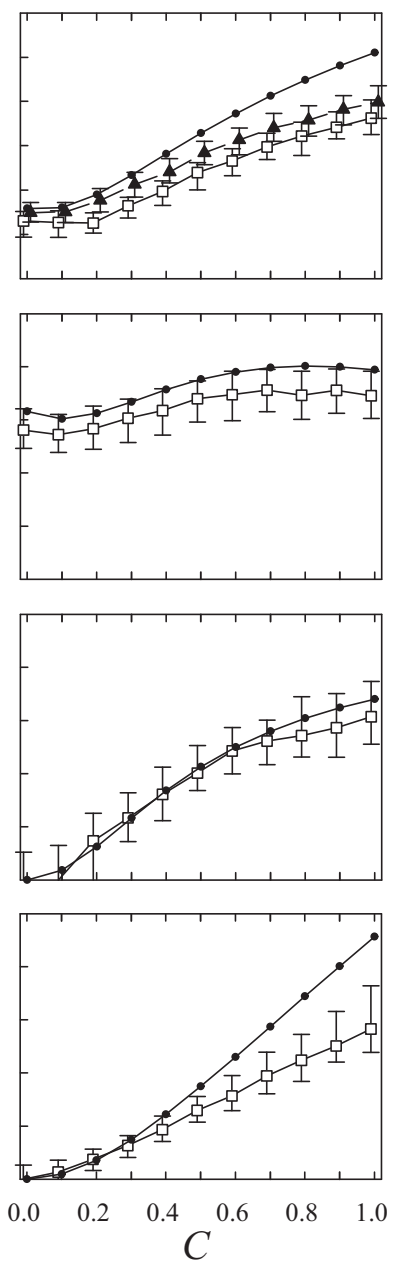

FIG. 3. Estimation of measures of information dynamics for simulation A (linear VAR process) with delay $\delta=10$. Plots and symbols are the same as in Fig. 2.

sets of MI (or entropies) involving vectors of dimension that differs much less than for PE, so the sum of the three biases is still smaller than the bias of the direct PE estimate.

\section{B. Coupled Hènon maps}

The second system considered was the nonlinear deterministic system with $M$ coupled Hénon maps studied in [30]:

$$
\begin{aligned}
X_{i, n}= & 1.4-X_{i, n-1}^{2}+0.3 X_{i, n-2}, \quad i=1, M \\
X_{i, n}= & 1.4-\left[0.5 C\left(X_{i-1, n-1}+X_{i+1, n-1}\right)+(1-C) X_{i, n-1}\right]^{2} \\
& +0.3 X_{i, n-2}, \quad i=2, \ldots, M-1,
\end{aligned}
$$

where the parameter $C$ was varied to modulate the coupling from the $(i+1)$ th and $(i-1)$ th systems towards the $i$ th system. We considered the cases of $M=5$ and 15 coupled systems, for which complete synchronization is not observed for any pair of systems. Moreover, since the time series of the driven system explode for $C>1$ [30], the analysis was performed by letting $C$ vary from 0 to 0.8 in steps of 0.1 .

The analysis was performed by considering the central map of the cascade as the destination process and taking either the 

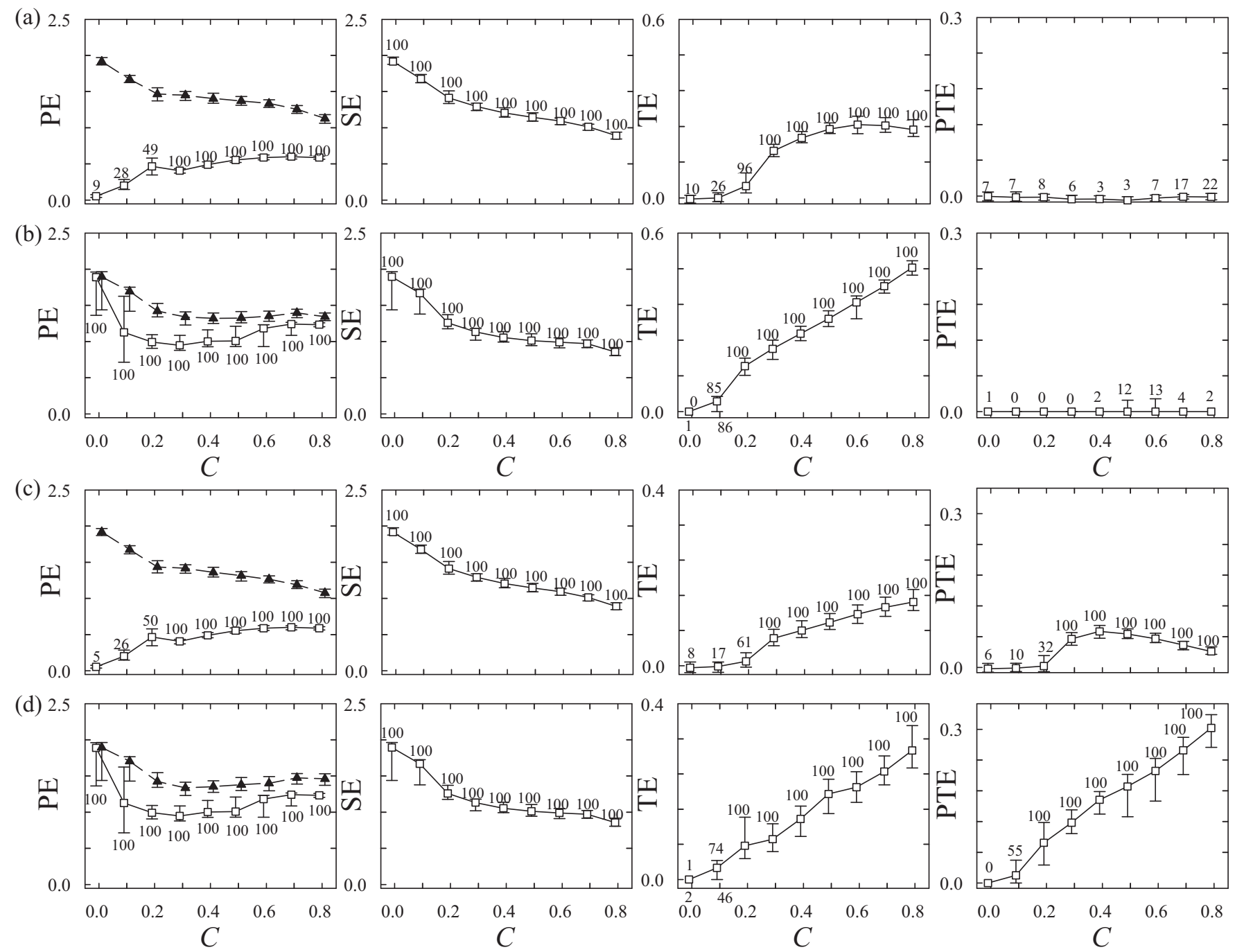

FIG. 4. Estimation of measures of information dynamics for simulation B (nonlinear Hénon maps) with $M=5$ processes, assuming the target process $Y=X_{3}$ and source process (a) and (b) $X=X_{1}$ or (c) and (d) $X=X_{2}$. Plots depict the estimated values (median and 10th-90th percentiles over 100 process realizations) of the PE of $Y$, SE of $Y$, TE from $Z$ to $Y$, and PTE from $X$ to $Y$ conditioned to $Z$, computed as a function of the coupling parameter $C$ by means of (a) and (c) the uniform embedding approach and (b) and (d) the nonuniform embedding approach. The number of realizations (out of 100) for which a measure was detected as statistically significant is reported close to its distribution. The Prediction Entropy is computed through the direct estimation (white squares) as well as the sum of the estimated SE, TE and PTE (black triangles).

closest not directly coupled map or the closest directly coupled map as the source process. Thus, we considered $Y=X_{3}$ together with $X=X_{1}$ or $X=X_{2}$ when $M=5$ and $Y=X_{8}$ together with $X=X_{6}$ or $X=X_{7}$ when $M=15$, collecting in each case all the remaining maps in the vector process Z. Uniform embedding was performed using $m_{X}=m_{Y}=$ $m_{Z}=1$ and $d_{X}=d_{Y}=d_{Z}=2$ as embedding parameters, while nonuniform embedding was performed using $L=5$ candidates for each process. To evaluate the sensitivity and the specificity in the estimation of the information dynamics, in this simulation we assessed also the statistical significance of each estimated PE, SE, TE, and PTE measure. The measures assessed by nonuniform embedding were considered as statistically significant whenever they resulted in being strictly positive as a consequence of the selection of at least one relevant component [i.e., at least one past component from the processes appearing only in the second conditional entropy term on the right-hand side of Eqs. (1) and (3)-(5)]. The statistical significance of measures assessed by uniform embedding was assessed through surrogate-data analysis, generating 40 time-shifted surrogates [42] and rejecting the null hypothesis of the absence of predictive information, storage, or transfer, respectively, if the original PE, TE, or PTE took the first or second position in the descending ordered sequence of original and surrogate values; according to the distribution-free plotting position formula given in [43], this corresponds to a significance level of $4.05 \%$.

The results of this simulation are depicted in Figs. 4 and 5. Similarly to what was observed for simulation A, we found that the PE estimates were generally lower when computed directly using Eq. (15) than when computed by summing up the SE, TE, and PTE estimates. Although theoretical values allowing exact bias inference are not available for this nonlinear system, the comparison between the two estimates of PE suggests 
(a)
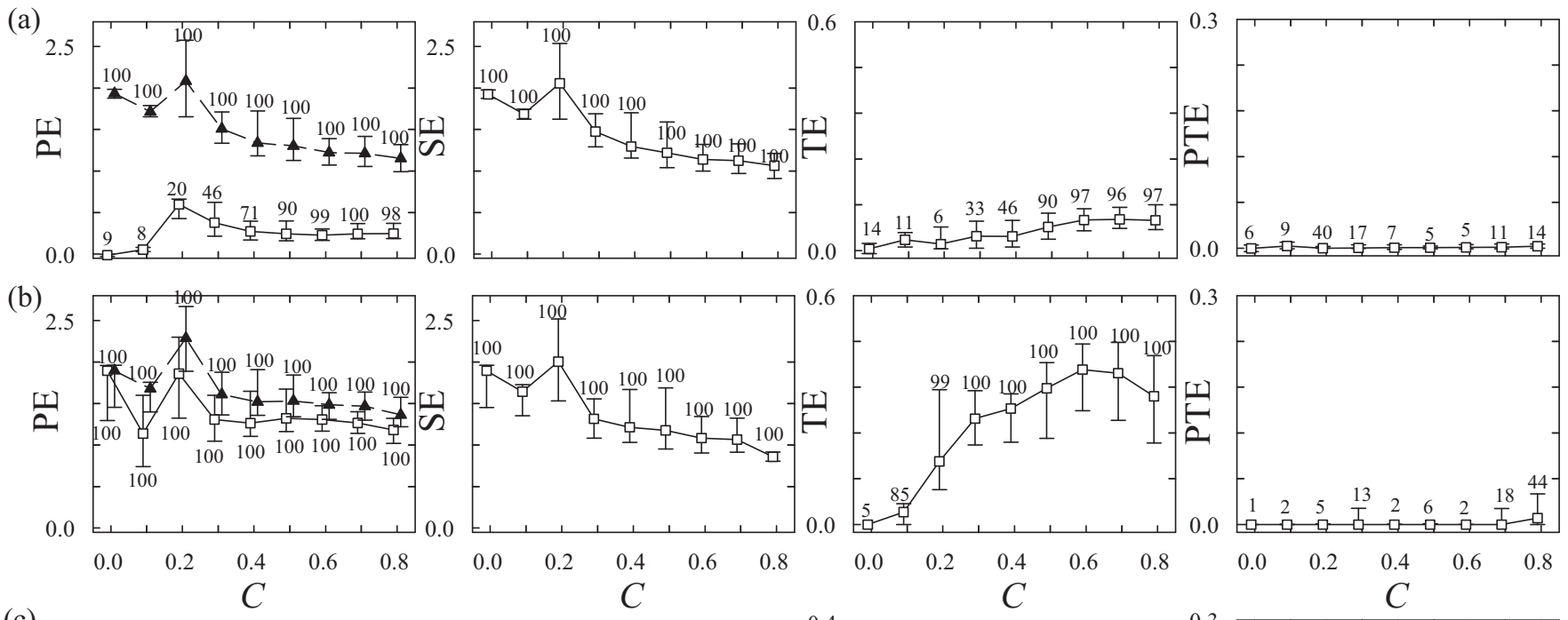

(c)
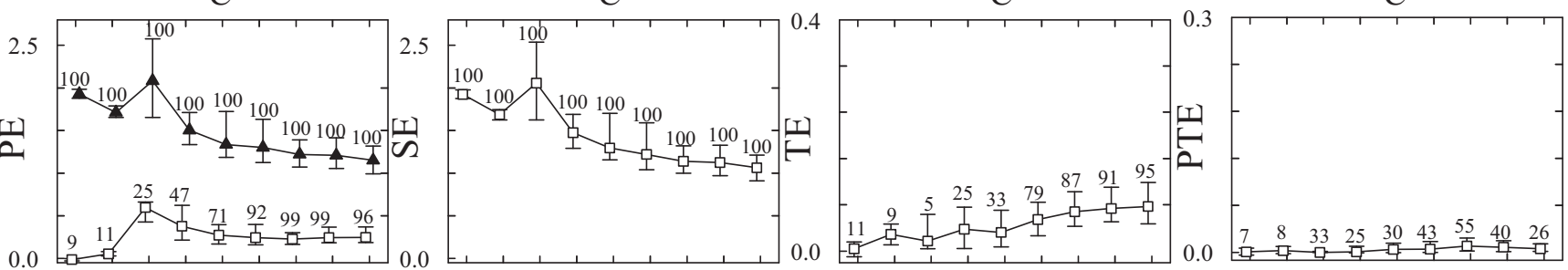

(d)
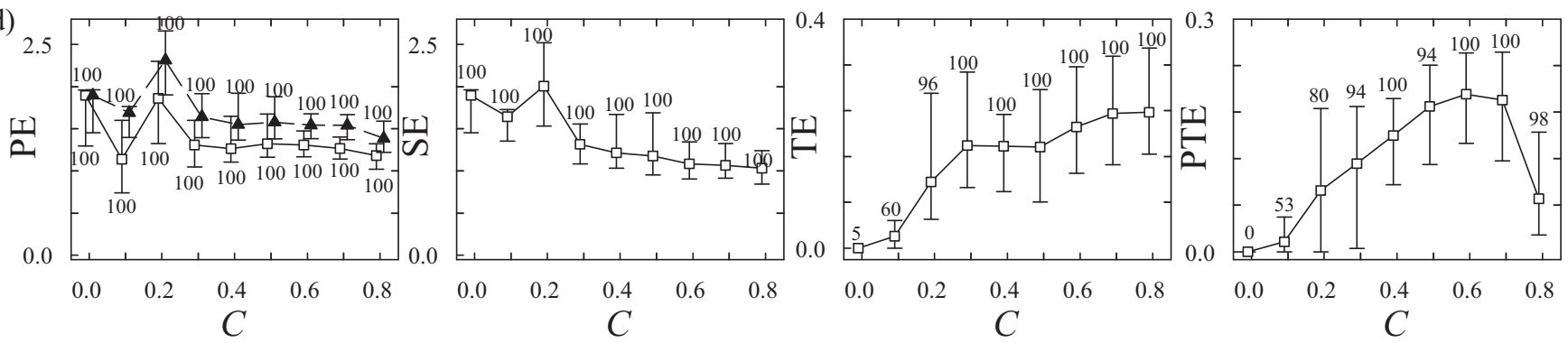

FIG. 5. Estimation of measures of information dynamics for simulation B (nonlinear Hénon maps) with $M=15$ processes, assuming the target process $Y=X_{8}$ and source process (a) and (b) $X=X_{6}$ or (c) and (d) $X=X_{7}$. Plots and symbols are the same as in Fig. 4.

that the nonuniform embedding scheme achieves a lower bias than uniform embedding in the estimation of the predictive information. This observation is supported by (i) the greater difference between the two estimates of PE using uniform embedding [Figs. 4(a), 4(c), 5(a), and 5(c)] and nonuniform embedding [Figs. 4(b), 4(d), 5(b), 5(d)] and (ii) the consistent trends in magnitude and statistical significance of the two PE estimates as a function of $C$ using nonuniform embedding [Figs. 4(b), 4(d), 5(b), and 5(d)], which were not achieved using uniform embedding [e.g., the two PE estimates in Figs. 4(a) and 4(c) did not show similar trends at increasing $C$ ].

The results shown in Fig. 4 regard the analysis with $M=5$ interacting processes and particularly the indirect coupling $X_{1} \rightarrow X_{3}$ [Figs. 4(a) and 4(b)] and the direct coupling $X_{2} \rightarrow$ $X_{3}$ [Figs. 4(c) and 4(d)], both absent when $C=0$ and present when $C>0$ with a strength related to $C$; in addition there is an autodependence of $X_{3}$ on its own past, always present but with decreasing strength as $C$ increases. Accordingly we expect, for the target process $Y=X_{3}$ to measure $P_{Y}$ as always significant, $S_{Y}$ as always significant and decreasing at increasing $C, T_{\mathbf{Z} \rightarrow Y}$ as significant for $C>0$ and increasing with $C$, and $T_{X \rightarrow Y \mid Z}$ as never significant when $X=X_{1}$ but significant for $C>0$ and increasing with $C$ when $X=X_{2}$. These expected results were fully verified only when the estimation was based on nonuniform embedding [Figs. 4(b) and 4(d)]: PE and SE were estimated as statistically significant in all conditions; TE was almost zero and insignificant for $C=0$ but significant and increasing with $C$ for $C>0$; PTE was insignificant for all values of $C$ (though with some false positives at $C=0.5$ and 0.6) in the absence of direct coupling [Fig. 4(b)] and well reflecting the coupling strength in the presence of direct coupling [Fig. 4(d). Using uniform embedding [Figs. 4(a) and 4(c)], we found that the PE was high and significant for all values of $C$ only when it was computed as the sum of SE, TE, and PTE estimated separately; the SE estimates were reliable; the TE and the PTE were also reliable, but showed a higher number of false positives and a less evident trend with increasing $C$ compared to the estimates yielded by nonuniform embedding.

The results of the analysis performed on simulations with $M=15$ processes are reported in Fig. 5. Since the coupling structure is preserved, the expected trends of the various information dynamics are the same as for the simulation 
with $M=5$. However, in this more challenging condition the estimation led to fewer interpretable results in the case of uniform embedding. In particular, using uniform embedding, we could not clearly detect the expected increase of the TE with $C$ [see Figs. 5(a) and 5(c), in which the response of both the magnitude and the statistical significance of $T_{\mathbf{Z} \rightarrow Y}$ to increasing $C$ was blunted] and even less the increase of the PTE with $C$ [see Fig. 5(c), in which $T_{X \rightarrow Y \mid Z}$ exhibited an almost flat profile with a limited number of realizations indicating statistical significance of the measure]. As can be seen in Figs. 5(b) and 5(d), the estimation improved with utilization of the nonuniform embedding scheme, as both the TE and the PTE were of higher magnitude (though with high variability across realizations) and the results of statistical significance evidenced high specificity and sensitivity of the measures (apart from the higher number of false positives estimated for $T_{X \rightarrow Y \mid \mathbf{Z}}$ with $C=0.8$ ).

\section{REAL DATA APPLICATIONS}

This section describes the evaluation of our framework for the estimation of information dynamics on real time series obtained from interacting physiological systems. The considered applications are the analysis of how short-term heart rate variability is generated from cardiovascular and cardiorespiratory interactions and the study of brain-heart interactions during sleep. These applications present challenging analysis conditions, as only short time series (a few hundred points) are made available due to stationarity constraints or finiteness of the measurable data sets. The analysis is focused on demonstrating the feasibility of information decomposition in terms of capability of the proposed estimators to detect statistically significant information dynamics and to describe expected physiological knowledge about the observed systems.

In real data applications, estimation of information dynamics measures was performed on normalized versions of the considered time series, using the following settings for the parameters: $k=10$ neighbors, $m_{X}=m_{Y}=m_{Z}=1$ and $d_{X}=d_{Y}=d_{Z}=5$ as embedding parameters (uniform embedding), $L=5$ as the maximum lag, and $N_{r}=100$ and $\alpha=0.05$ for candidate component selection (nonuniform embedding). As in the simulations, the statistical significance of each measure was assessed when the measure was strictly positive for nonuniform embedding and using time-shifted surrogates for uniform embedding.

\section{A. Cardiovascular and cardiorespiratory variability}

In the first application we study cardiovascular and cardiorespiratory variability measured in healthy subjects during a head-up tilt testing protocol [44]. The protocol consisted in simultaneously monitoring the electrocardiogram (ECG), the finger photoplethysmographic arterial pressure, and the respiratory airflow in 15 subjects, both lying in the resting supine position and standing in the upright position reached by passive head-up tilting. The beat-to-beat variability series of the heart period (HP), the systolic arterial pressure (SAP), and the respiratory activity (RA) were measured, respectively, as the consecutive heartbeat interval durations from the ECG, the local maxima of the pressure signal considered between consecutive heartbeats, and the values of the respiration signal sampled at each heartbeat. For each subject and body position, stationary time series of 300 points were made available for the analysis. Weak stationarity was assessed by means of a test checking the stability of the mean and variance of the series over the analysis window [45]. We then considered the cardiac, vascular, and respiratory physiological systems as dynamical systems and let them be characterized by the HP variability (process $Y$ ), SAP variability (process $X$ ), and RA variability (process $Z$ ). Note that, since the time series are measured in a way such that the occurrence of the $n$th RA precedes in time the occurrence of the $n$th SAP and both occur before the end of the $n$th HP, the zero-lag terms $X_{n}$ and $Z_{n}$ were considered in the embedding schemes adopted to describe the target variable $Y_{n}$ [7].

In this application, utilizing the decomposition strategy detailed in Eqs. (1)-(5), the predictive information about the cardiac system, measured in terms of HP variability, is expressed as the sum of three meaningful contributions reflecting the information dynamically stored in the cardiac system and that transferred to the system from the respiratory and vascular systems. Measuring how much of the information about the present HP can be predicted from its past, the SE is a quantity closely related to several complexity indexes measuring how much of the information about the present cannot be predicted from the past, which have been fruitfully used to characterize the short-term complexity of heart rate variability, particularly in head-up tilt test protocols [46]. Additionally, the $\mathrm{TE} T_{Z \rightarrow Y}$ reflects the amount of information provided to the present HP by the past of RA that was not already provided by the past of HP, thus accounting for all (both direct and mediated by SAP) respiratory effects on cardiac variability [47]. Then the PTE $T_{X \rightarrow Y \mid \mathbf{Z}}$ quantifies the direct effects of the past of SAP on the present of HP; in this case, conditioning on the past of RA is advisable to rule out spurious information transfers likely arising from the common driver effect of RA on SAP and HP [48].

Estimation of all measures on the 15 subjects considered led to the results shown in Fig. 6, where the statistical significance of the difference between the distributions of a given measure in the supine and upright positions was assessed using the student's $t$-test for paired data. As shown in the figure, detectable amounts of predictive information, information storage, and information transfer were observed in almost all subjects. The PE and the SE were significant for all subjects in both conditions independently of the method used for their estimation, indicating that a significant part of the information carried by HP variability can be explained from the knowledge of its own past. In the supine position, the TE from RA to HP was also always significant, documenting the existence of an important cardiorespiratory information transfer that likely reflects the well-known phenomenon of respiratory sinus arrhythmia [49]. On the other hand, the existence of a significant PTE from SAP to HP conditioned to RA was less evident. However, this result is consistent with the observation that the related mechanism, i.e., the baroreflex information transfer from SAP to HP, seems not to be fully solicited in the resting supine position [50]. With the transition to the upright position, the predictive information increased significantly, mainly as a result of the substantial increase of the information storage. The higher SE observed after tilt is in line with several 

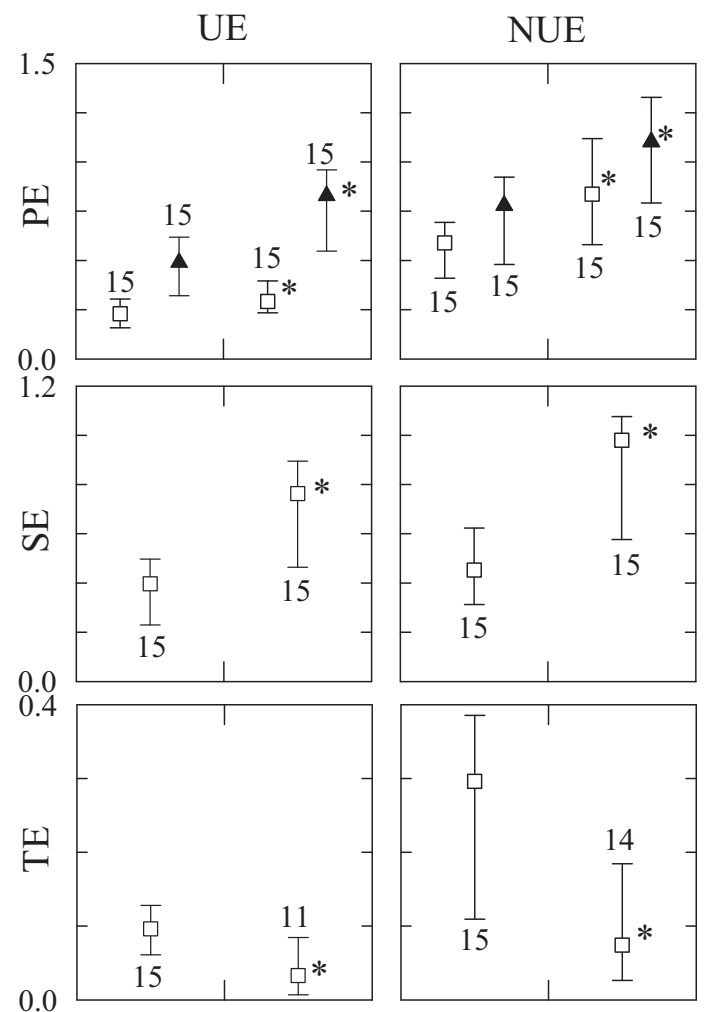

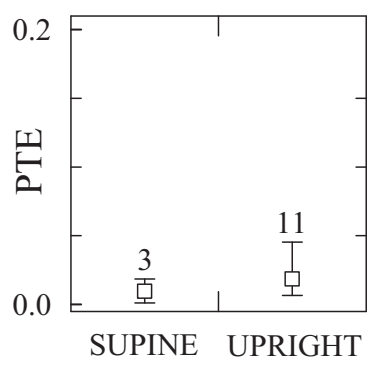

(a)

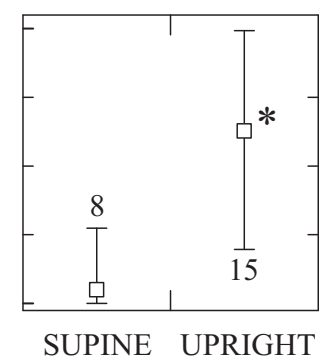

(b)
FIG. 6. Estimation of measures of information dynamics for the first application $(X$, systolic arterial pressure; $Y$, heart period; and $Z$, respiratory activity). Plots depict the estimated values (median and 10th-90th percentiles over 15 subjects) of the PE of $Y$, SE of $Y$, TE from $Z$ to $Y$, and PTE from $X$ to $Y$ conditioned to $Z$, computed in the supine position and upright position by means of (a) uniform embedding and (b) nonuniform embedding. The Prediction Entropy is computed through the direct estimation (squares) as well as the sum of the estimated SE, TE and PTE (triangles). The number of subjects (out of 15) for which any measure was detected as statistically significant is reported close to its distribution. Asterisks indicate statistically significant differences of supine vs upright (paired $t$-test, $p<0.05$ ).

previous findings that demonstrated that standing decreases the complexity of HP variability; this decrease in complexity, here detected as an increase of information storage, has been related to the tilt-induced activation of the sympathetic nervous system and corresponding deactivation of the vagal parasympathetic system $[13,46]$. The shift of the sympathovagal balance towards its sympathetic branch also likely explains both the significant reduction of the TE from RA to HP and the significant increase of the PTE from SAP to HP conditioned to RA, observed in the upright position compared to the supine. Indeed, vagal deactivation is known to be associated with dampened respiratory sinus arrhythmia [49], which in our protocol is reflected by a weakened cardiorespiratory coupling. Moreover, the sympathetic activation induced by tilt is known to solicit the heart rate response to arterial pressure changes [51,52], which here is reflected in terms of enhanced information transfer through the baroreflex.

The above physiological interpretations were better supported by the results obtained using the nonuniform embedding approach. Indeed, uniform embedding generally provided lower estimates of information dynamics, which in some cases prevented the detection of expected changes; an example is the increase of information transfer from SAP to HP that was detected as statistically significant only when the PTE was assessed through nonuniform embedding. Moreover, similarly to what was observed for simulated data, the difference between the two available PE estimates was more marked when using uniform embedding compared to the nonuniform scheme, suggesting a higher bias. These results indicate that the proposed nonuniform embedding scheme should be adopted for a more efficient estimation of information dynamics in real physiological time series.

\section{B. Electroencephalogram-heart rate interactions during sleep}

In the second application we study interactions between the time series of brain wave activities and cardiac vagal autonomic activity and measured during sleep [53]. In this experiment, polysomnography was performed in ten young healthy male subjects, measuring electroencephalogram (EEG) and ECG activity during the whole night. Five time series related to brain wave activity were obtained by computing the spectral power inside the conventional EEG bands $(\delta, 0.5-3 \mathrm{~Hz} ; \theta$, $3-8 \mathrm{~Hz} ; \alpha, 8-12 \mathrm{~Hz} ; \sigma, 12-16 \mathrm{~Hz}$; and $\beta, 16-25 \mathrm{~Hz}$ ) for consecutive 5-s windows and then averaging every $60 \mathrm{~s}$ and normalizing to the full night mean power in the band. At the same time, the time series of cardiac vagal activity was obtained by evaluating the spectral power of the heartbeat intervals in the high-frequency $(\mathrm{HF})$ band $(0.15-0.4 \mathrm{~Hz})$, normalized to the total power in the range $0.04-0.4 \mathrm{~Hz}$, for consecutive 120-s windows overlapped by half. The whole night analysis produced time series of length between 440 and 520 points. Details about experimental protocol and time series measurement can be found in [53]. In this study we consider the EEG band powers as the processes describing the dynamics of the five subsystems that are thought of as constituents of the brain system and the cardiac HF power as the process describing the dynamics of the heart system. Here the analysis of brain-heart interactions is focused on the relation between EEG $\delta$ wave power and cardiac HF power, which has been the subject of several previous studies about brain-heart interactions [53-56]. Accordingly, we assume the EEG $\delta$ power and the HF power as source and target processes $X$ and $Y$, investigating both directions of interaction, and collect the remaining four EEG powers in the process $\mathbf{Z}$.

Figure 7 reports the results of the analysis performed over all subjects taking either the EEG $\delta$ power or the cardiac HF power as the target process. In either case, both the PE and SE resulted in being statistically significant independently of the adopted estimator, documenting the existence of structured 


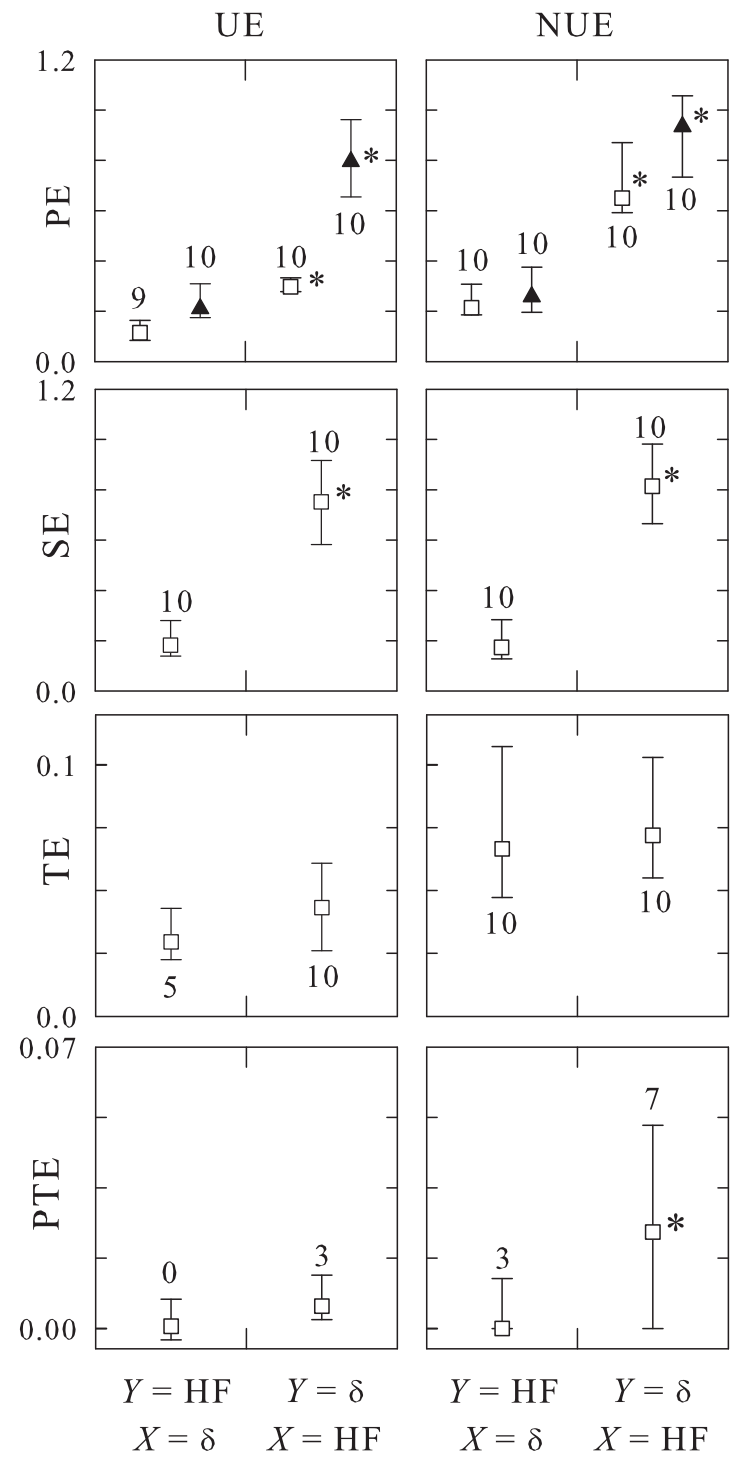

(a)

FIG. 7. Estimation of measures of information dynamics for the second application (time series of EEG and cardiac HF variability band powers). Plots depict the estimated values (median and 10th90th percentiles over 10 subjects) of the PE of $Y, \mathrm{SE}$ of $Y$, TE from $\mathbf{Z}$ to $Y$, and PTE from $X$ to $Y$ conditioned to $\mathbf{Z}$, computed by means of (a) uniform embedding and (b) nonuniform embedding. The analysis is performed by taking the cardiac HF activity as the target process and the EEG $\delta$ power as the source process and vice versa. The Prediction Entropy is computed through the direct estimation (squares) as well as the sum of the estimated SE, TE and PTE (triangles). The number of subjects (out of 10) for which any measure was detected as statistically significant is reported close to its distribution. Asterisks indicate statistically significant differences between the two directions of interaction (paired $t$-test, $p<0.05$ ).

dynamics in the processes descriptive of cardiac HF and brain $\delta$ activities and indicating that a significant part of these dynamics can be expressed in terms of information storage. The analysis of TE computed with the EEG $\delta$ activity as the target process demonstrated the existence of significant information transfer directed towards the $\delta$ node of the brain-

brain subnetwork ( $T_{\mathbf{Z} \rightarrow Y}$ was substantial and significant in all subjects). This result agrees with the findings of a recent study conducted on the same data set using a linear model-based estimator [57], where we more specifically found that there is significant information transferred to each node of the brain-brain network composed of the $\delta, \theta, \alpha, \sigma$, and $\beta$ EEG wave amplitudes. Taking the cardiac HF power as the target process, we found high and significant TE values, indicating an important transfer of information from the brain system to the heart system. This result, which was fully evident only using nonuniform embedding estimators, supports previous studies conducted on the same data, based on linear frequency domain analysis [53] and nonlinear synchronization analysis [56], which showed that all EEG power band dynamics are linked to cardiac HF variability. Additionally, our decomposition shows that the important brain-to-heart transfer, documented by the significant $\mathrm{TE} T_{\mathbf{Z} \rightarrow Y}$, is mediated only in a small part by the $\delta$ activity because the corresponding PTE $T_{X \rightarrow Y \mid \mathbf{Z}}$ is very low and barely significant. On the other hand, the PTE estimated through nonuniform embedding over the opposite direction from cardiac HF activity to EEG $\delta$ activity was significantly higher ( $p<0.05$ according to the paired $t$-test) and detected in a higher number of subjects ( 7 vs 3 ). This result suggests that information is exchanged between the brain and heart systems along the preferential direction going from cardiac to $\delta$ wave activity and is in line with previous findings reporting that the oscillations of the EEG $\delta$ power lag behind those of the cardiac HF time series during sleep [53]. The inability of the traditional uniform embedding to detect this expected direction for brain-heart interactions suggests once more the better suitability of nonuniform embedding schemes for the detection of predictive information transfer in real data measured from physiological systems. Nevertheless, the different complexity of the EEG $\delta$ amplitude and the cardiac HF component, here documented by the different SE values, may play a role in determining the asymmetry of the information transfer between these two series. Also, an important limitation of the adopted acquisition protocol is that it did not include the respiratory signal. Since respiration affects both the EEG power and heart rate variability, it may be the case that the observed information transfer is driven by the common effects of the unobserved respiratory variable. Future studies should be directed to applying the proposed fully multivariate framework to more complete data sets including potentially confounding variables.

\section{DISCUSSION}

The present study underlines the need to resort to approaches for an accurate selection of the variables to be included in the entropy terms composing the measures of information dynamics, when these measures have to be estimated under conditions requiring the exploration of state spaces of high dimension. Under these conditions, typically occurring in the analysis of multiple interacting processes with long memory, the curse of dimensionality becomes a severe issue. In fact, this issue has limited the practical development of fully multivariate approaches to the estimation of dynamical information-theoretic measures. Only recently have several approaches for dimensionality reduction 
been proposed specifically for the evaluation of multivariate measures of direct coupling such as Granger causality or the PTE $[9,30,40,58]$. The approach proposed in this study generalizes the nonuniform embedding scheme introduced in [30] to the estimation of the PE and of the factors of its decomposition. With this approach, reduction of the dimension of the state space to a minimum value is achieved by applying an optimization criterion, based on CMI maximization, such that only the past process components that contribute most (in terms of CMI) to the present of the target process are included in the computation. The most distinctive features of the approach are that (i) it employs a nearest-neighbor estimation technique specifically designed to compensate the bias due to the different dimensionality of individual entropy terms [4,29]; (ii) it incorporates an automatic termination criterion for the selection of components, which allows bypassing the problem of determining the embedding parameters; and (iii) it returns values of PE, SE, TE, and PTE that are strictly positive only when associated with significant predictive information, information storage, or information transfer and exactly zero otherwise. The latter property, which results from the randomization test applied to assess the significance of each selected component, adds to the output measure the important information about its statistical significance, thus avoiding the need of performing time-consuming significance tests after the estimation. Therefore, while our approach is slower than the traditional uniform embedding, it becomes less computationally intensive when the statistical significance of the considered information measure needs to be assessed. Moreover, although this feature has not been exploited here, the selection of significant lagged components achieved by nonuniform embedding allows us also to infer the time lags at which the causal dependences take place [41].

In general, it is known that the estimation of informationtheoretic measures is hampered by several problems other than the curse of dimensionality, including the estimation bias resulting from working on limited amounts of possibly noisy data, the different bias brought by the estimation of the different terms composing the investigated measure, and the different internal dynamics of each investigated system [26,27,29]. The most general measure considered in this study, i.e., the predictive information, reflects the overall statistical dependence of the present state of the observed target system on the past history of the whole observed multivariate system. Being expressed as the sum of different terms that may contribute with different bias to the predictive information, its direct estimation may not support the validity of tiny changes in the composing terms, especially when they are of low magnitude. We have shown that this issue is better addressed by providing individual estimates of SE, TE, and PTE and using their sum to estimate the PE. Moreover, it is known that inference of the causal relations, in this context performed through the measures of information transfer, is not a trivial issue as it is complicated by issues related to differences in the estimation bias of the entropy estimates and in the internal complexity of the observed source and target processes [26]. These issues may have an impact on causality estimation, as they easily lead to decreased sensitivity and false detections. A major aim of the framework for the estimation of information dynamics presented in this study was that of limiting such an impact.
Our results suggest that our approach addresses successfully the problem of quantifying the predictive information about a target process embedded in a network of interacting processes, as well as the information storage and transfer terms that form its decomposition, even in the challenging conditions posed by the presence of several processes and/or long interaction delays. In these conditions, the good statistical properties and the bias compensation provided by the nearest-neighbor estimation method are not sufficient to make it able to assess reliably the values of PE, SE, TE, and PTE if it is implemented according to the classical uniform embedding scheme. In contrast, implementation of the nearest-neighbor estimator in the proposed nonuniform embedding procedure leads to an accurate detection of the significant values of all measures of information dynamics and allows containing the bias of their estimated magnitude. This has been documented by both comparing expected and estimated values of the measures in the simulation of linear Gaussian processes (Figs. 2 and 3) and assessing the statistical significance of the estimated measures in the simulation of nonlinear chaotic systems (Figs. 4 and 5). The good sensitivity and specificity of nonuniform embedding was also previously demonstrated for the task of causality detection [30,59]. Of note is that the good performance has been achieved in this study on very short process realizations (a few hundred data points). This is important for the analysis of real-world time series, for which the dynamics typically unfold over short periods of time.

The feasibility of estimating predictive information and its decomposition in real data has been demonstrated by the two applications, in which the analysis of information dynamics led to physiologically interpretable results. In particular, in the cardiovascular application where the adopted experimental protocol allowed us to establish a sort of verifiable ground truth, the expected increase of information storage in the cardiac system, decrease of cardiorespiratory information transfer, and increase in cardiovascular transfer were all detected at the transition from supine to upright body position (Fig. 6). Also in the study of cardiac and EEG time series during sleep, the observed patterns of brain-brain and brainheart interactions were in line with the results of previous experimental findings.

From a more theoretical point of view, we confirm the importance of adopting a complete analysis framework whereby measures of information storage and transfer are computed and interpreted together as constituents of the predictive information about a dynamical system embedded in a network of interacting systems [23]. In our study, the usefulness of a combined analysis can be inferred, for instance, by looking at Figs. 4 and 5, where the tendency towards a decrease in the predictive information estimated for increasing coupling parameter is explained by a decrease in the information stored in the target process, not fully compensated by the increasing information transfer. Another interesting behavior is that displayed by the information storage in Figs. 2 and 3: While one would expect the SE to decrease with the coupling parameter [given that a higher $C$ in Eq. (16) determines less strong internal dynamics in the target process], the nonmonotonic behavior of SE results from the knowledge that information storage can be both internally driven and inputdriven [5] and from the observation that in this example the 
information transfer increases substantially with the coupling parameter. The need to provide a joint interpretation of the different measures of information dynamics is supported also by our real data analysis. For example, in Fig. 6, the increase of the predictive information about the cardiac system observed going from the supine to the upright position is reflected by variations of different sign, all physiologically well interpretable, of the measures of information storage and transfer: the SE increases reflecting sympathetic activation [46], the TE decreases reflecting a dampening of the respiratory sinus arrhythmia [18], and the PTE increases reflecting an activation of the cardiac baroreflex [52].

Finally, it is worth noting that the decomposition of the predictive information is not unique: The implicit arbitrariness in the entropy decomposition rule allows decomposing the PE following a different ordering, when progressively conditioning on the past of the processes, than that presented in Eq. (2). While from a theoretical point of view none of the possible decompositions may be considered as preeminent [23,60], in this study we adopted the one in Eq. (2) because it puts in evidence well-defined quantities such as the SE, the TE, and the PTE. Here this decomposition is also supported $a$ posteriori by our applicative analyses showing how it helps in understanding the dynamic dependences in different networks of physiological systems. Nevertheless, the estimation framework proposed in Sec. III can be intuitively implemented to support other decompositions when they are considered more appropriate. In addition, it is worth noting that the predictive information terms computed for all possible target processes form the so-called global multi-information [23], which is a measure of the whole temporal statistical dependences for a multivariate process. Since the global multi-information can be alternatively decomposed evidencing amounts of information related to the temporal internal dynamics of each process and the directed transfer of information between processes (plus instantaneous exchange of information) [23,61,62], the predictive information decomposition presented in this study can be placed in a more general context where its terms characterize distinctive parcels of the overall causal statistical structure of networks of dynamic processes.

\section{ACKNOWLEDGMENTS}

This work was supported in part by the Healthcare Research Implementation Program (IRCS), Provincia Autonoma di Trento and Bruno Kessler Foundation, Italy and by the University of Gent (Special Research Funds for visiting researchers).

\section{APPENDIX}

Here we provide a derivation of the exact values of the measures of information dynamics when the observed $M$-dimensional process $\boldsymbol{U}=\{X, Y, \boldsymbol{Z}\}$ has a joint Gaussian distribution. The derivation is based on first expressing the joint Gaussian process as a VAR process, for which the autocovariance sequence can be computed, up to arbitrarily large lags, from the VAR parameters and then exploiting the equations that express PE, SE, TE, and PTE of joint Gaussian processes in terms of the cross covariances between their time-ordered variables.
Under the joint Gaussian assumption, it has been formally proven [35] that the statistical dependences between the present and the past variables constituting the process $\boldsymbol{U}$ are fully represented in terms of linear functions and thus can be properly accounted for by expressing $\boldsymbol{U}$ as a VAR process of appropriate order $p$ :

$$
\boldsymbol{U}_{n}=\sum_{k=1}^{p} \mathbf{A}_{k} \boldsymbol{U}_{n-k}+\boldsymbol{\varepsilon}_{n}
$$

where $\boldsymbol{U}_{n}=\left[\begin{array}{lll}X_{n} & Y_{n} & \boldsymbol{Z}_{n}\end{array}\right]^{\mathrm{T}}$ is the $M \times 1$ vector including the present variables of the joint process, $\mathbf{A}_{k}$ are $M \times M$ coefficient matrices, and $\boldsymbol{\varepsilon}_{n}$ is an $M \times 1$ white noise process with diagonal covariance matrix $\Lambda$. The autocovariance of the process (A1) is a sequence of $M \times M$ matrices $\boldsymbol{\Gamma}_{k}$ that are related to the VAR parameters via the Yule-Walker equations [63]

$$
\boldsymbol{\Gamma}_{k}=\boldsymbol{E}\left[\boldsymbol{U}_{n} \boldsymbol{U}_{n-k}^{T}\right]=\sum_{l=1}^{p} \mathbf{A}_{l} \boldsymbol{\Gamma}_{k-l}+\delta_{k 0} \boldsymbol{\Lambda},
$$

where $\delta_{k 0}$ is the Kronecker product. In order to solve (A2) for $k=0,1, \ldots, p-1$, we first express (A1) as

$$
\boldsymbol{U}_{n}^{p}=\mathbf{A}^{p} \boldsymbol{U}_{n-1}^{p}+\boldsymbol{\varepsilon}_{n}^{p},
$$

where

$$
\begin{aligned}
\boldsymbol{U}_{n}^{p} & =\left[\boldsymbol{U}_{n}^{T} \boldsymbol{U}_{n-1}^{T} \cdots \boldsymbol{U}_{n-p+1}^{T}\right]^{T}, \\
\mathbf{A}^{p}= & {\left[\begin{array}{llcc}
\mathbf{A}_{1} & \cdots & \mathbf{A}_{p-1} & \mathbf{A}_{p} \\
\mathbf{I}_{M \times M} & \cdots & \mathbf{0}_{M \times M} & \mathbf{0}_{M \times M} \\
\vdots & \ddots & \vdots & \vdots \\
\mathbf{0}_{M \times M} & \cdots & \mathbf{I}_{M \times M} & \mathbf{0}_{M \times M}
\end{array}\right], } \\
\boldsymbol{\varepsilon}_{n}^{p}= & {\left[\boldsymbol{\varepsilon}_{n}^{T} \mathbf{0}_{1 \times M(p-1)}\right]^{T}, }
\end{aligned}
$$

Then we find that the $p M \times p M$ covariance matrix of $\boldsymbol{U}_{n}^{p}$ is of the form

$$
\boldsymbol{\Gamma}_{0}^{p}=\boldsymbol{E}\left[\boldsymbol{U}_{n}^{p} \boldsymbol{U}_{n}^{p^{T}}\right]=\left[\begin{array}{lclc}
\boldsymbol{\Gamma}_{0} & \boldsymbol{\Gamma}_{1} & \cdots & \boldsymbol{\Gamma}_{p-1} \\
\boldsymbol{\Gamma}_{1}^{T} & \boldsymbol{\Gamma}_{0} & \cdots & \boldsymbol{\Gamma}_{p-2} \\
\vdots & \vdots & \ddots & \vdots \\
\boldsymbol{\Gamma}_{p-1}^{T} & \boldsymbol{\Gamma}_{p-2}^{T} & \cdots & \boldsymbol{\Gamma}_{0}
\end{array}\right]
$$

and can be derived by solving the equation

$$
\boldsymbol{\Gamma}_{0}^{p}=\mathbf{A}^{p} \boldsymbol{\Gamma}_{0}^{p} \mathbf{A}^{p T}+\boldsymbol{\Gamma}^{p},
$$

where $\boldsymbol{\Lambda}^{p}$ is the covariance of $\boldsymbol{\varepsilon}_{n}^{p}$, equal to

$$
\boldsymbol{\Lambda}^{p}=\boldsymbol{E}\left[\boldsymbol{\varepsilon}_{n}^{p} \boldsymbol{\varepsilon}_{n}^{p^{T}}\right]=\left[\begin{array}{cc}
\boldsymbol{\Lambda} & \mathbf{0}_{M \times M(p-1)} \\
\mathbf{0}_{M(p-1) \times M} & \mathbf{0}_{M(p-1) \times M(p-1)}
\end{array}\right] .
$$

Equation (A3) is a discrete-time Lyapunov equation, which can be solved for $\boldsymbol{\Gamma}_{0}^{p}$, thus yielding the values of $\boldsymbol{\Gamma}_{0}, \ldots, \boldsymbol{\Gamma}_{p-1}$. Then the autocovariance matrices can be calculated recursively for any $k \geqslant p$ by solving (A2) for $\boldsymbol{\Gamma}_{k}$ from the known $\Gamma_{k-1}, \ldots, \Gamma_{k-p}$. With this we have shown how to compute the autocovariance sequence of a VAR process, up to arbitrarily high lags, starting from the VAR parameters $\left(\mathbf{A}_{1}, \ldots, \mathbf{A}_{p}, \boldsymbol{\Lambda}\right)$. In the following we show how the elements of $\boldsymbol{\Gamma}_{k}$ can be exploited to calculate the information dynamics measures. 
Under the joint Gaussian assumption, the entropy of the target variable $Y_{n}$, the MI between $Y_{n}$ and the $k$-dimensional vector variable $V_{n}^{k}$, and the MI between $Y_{n}$ and $V_{n}^{k}$ conditioned to the $l$-dimensional vector variable $W_{n}^{l}$ can be formulated as $[34,64]$

$$
\begin{aligned}
H\left(Y_{n}\right) & =\frac{1}{2} \ln \left[2 \pi e \sigma\left(Y_{n}\right)\right], \\
I\left(Y_{n} ; V_{n}^{k}\right) & =\frac{1}{2} \ln \frac{\sigma\left(Y_{n}\right)}{\sigma\left(Y_{n} \mid V_{n}^{k}\right)}, \\
I\left(Y_{n} ; V_{n}^{k} \mid W_{n}^{l}\right) & =\frac{1}{2} \ln \frac{\sigma\left(Y_{n} \mid W_{n}^{l}\right)}{\sigma\left(Y_{n} \mid V_{n}^{k} \oplus W_{n}^{l}\right)},
\end{aligned}
$$

where $\sigma\left(Y_{n}\right)$ is the variance of $Y_{n}$ and $\sigma\left(Y_{n} \mid V_{n}^{k}\right)$ is the partial variance of $Y_{n}$ given $V_{n}^{k}$, that is, the variance of the residuals of a linear regression of $Y_{n}$ on $V_{n}^{k}$. The generic partial variance $\sigma\left(Y_{n} \mid V_{n}^{k}\right)$ can be expressed in terms of covariance matrices as [34]

$$
\sigma\left(Y_{n} \mid V_{n}^{k}\right)=\sigma\left(Y_{n}\right)-\Sigma\left(Y_{n}, V_{n}^{k}\right) \Sigma\left(V_{n}^{k}\right)^{-1} \Sigma\left(Y_{n}, V_{n}^{k}\right)^{T},
$$

with $\Sigma(\cdot)$ and $\Sigma(\cdot, \cdot)$ indicating, respectively, covariance and cross-covariance matrix. Therefore, $P_{Y}$ and the terms of its decompositions (i.e., $S_{Y}, T_{Z \rightarrow Y}$, and $T_{X \rightarrow Y \mid Z}$ ) can all be obtained: (i) letting $V_{n}^{k}$ and $W_{n}^{l}$ be the appropriate vector variables including the lagged components taken from the past of the observed processes $X_{n}^{-}, Y_{n}^{-}$and $\mathbf{Z}_{n}^{-}$; (ii) computing the relevant covariances to be used in (A5), which contain as scalar elements the covariance between lagged components of $X, Y$, and $\mathbf{Z}$ and as such can be extrapolated by proper arrangement of the elements of the autocovariance sequence $\boldsymbol{\Gamma}_{k}$ of the overall process $\boldsymbol{U}$; and (iii) solving (A5) to get the partial variances to be inserted in (A4). For practical computation, the infinite-dimensional vectors $X_{n}^{-}, Y_{n}^{-}$, and $\boldsymbol{Z}_{n}^{-}$ are truncated at dimension $L: X_{n}^{L}=\left[X_{n-1} X_{n-2} \cdots X_{n-L}\right]$, $Y_{n}^{L}=\left[Y_{n-1} Y_{n-2} \cdots Y_{n-L}\right]$, and $\boldsymbol{Z}_{n}^{L}=\left[\boldsymbol{Z}_{n-1} \oplus \boldsymbol{Z}_{n-2} \oplus \cdots \oplus\right.$ $\left.Z_{n-L}\right]$. For instance, the PTE defined in (5) is computed through the last equation in (A4) after setting $V_{n}^{k}=X_{n}^{L}$ and $W_{n}^{l}=Y_{n}^{L} \oplus \boldsymbol{Z}_{n}^{L}$ [with $k=L$ and $l=(M-2) L$ ]. As regards the optimal truncation for the past history of the processes, we note that considering the past up to lag $L$ corresponds to calculating the autocovariance of the process (A1) up to the matrix $\boldsymbol{\Gamma}_{L}$. Since the autocovariance of a VAR process decays exponentially with the lag, with a rate of decay depending on the spectral radius of the process $\rho(A)$ (which is just the modulus of the largest eigenvalue of $\boldsymbol{A}^{p}$ ), it has been suggested as a rule of thumb to compute the autocovariance up to a lag $L$ such that $\rho(A)^{L}$ is smaller than a predefined numerical tolerance [e.g., $10^{-8}$, which can lead to very high lags as the VAR approaches the unstable regime $\rho(A) \rightarrow 1$ ] [63]. Nevertheless, after trying different VAR configurations, we found that the computation of very long autocovariance sequences is not necessary for the purpose of evaluating information dynamics because all measures stabilize to constant values already for small lags (typically $L=10$ ) even for reasonably high values of the spectral radius [47].
[1] J. T. Lizier, The Local Information Dynamics of Distributed Computation in Complex Systems (Springer, Berlin, 2013).

[2] J. T. Lizier, M. Prokopenko, and A. Y. Zomaya, Inf. Sci. 208, 39 (2012).

[3] T. Schreiber, Phys. Rev. Lett. 85, 461 (2000).

[4] R. Vicente, M. Wibral, M. Lindner, and G. Pipa, J. Comput. Neurosci. 30, 45 (2011).

[5] M. Wibral, J. T. Lizier, S. Vogler, V. Priesemann, and R. Galuske, Front. Neuroinf. 8, 1 (2014).

[6] J. Lee, S. Nemati, I. Silva, B. A. Edwards, J. P. Butler, and A. Malhotra, Biomed. Eng. Online 11, 19 (2012).

[7] L. Faes, G. Nollo, and A. Porta, Entropy 15, 198 (2013).

[8] D. Kugiumtzis, Eur. Phys. J. 222, 401 (2013).

[9] J. Runge, J. Heitzig, V. Petoukhov, and J. Kurths, Phys. Rev. Lett. 108, 258701 (2012).

[10] S. M. Pincus, Proc. Natl. Acad. Sci. U.S.A. 88, 2297 (1991).

[11] J. S. Richman and J. R. Moorman, Am. J. Physiol. Heart Circ. Physiol. 278, H2039 (2000).

[12] A. Porta, G. Baselli, D. Liberati, N. Montano, C. Cogliati, T. Gnecchi-Ruscone, A. Malliani, and S. Cerutti, Biol. Cybern. 78, 71 (1998).

[13] L. Faes, A. Porta, G. Rossato, A. Adami, D. Tonon, A. Corica, and G. Nollo, Auton. Neurosci. 178, 76 (2013).

[14] C. W. J. Granger, Econometrica 37, 424 (1969).

[15] M. Wibral, B. Rahm, M. Rieder, M. Lindner, R. Vicente, and J. Kaiser, Prog. Biophys. Mol. Biol. 105, 80 (2011).

[16] T. Dimpfl and F. J. Peter, Stud Nonlinear Dyn E 17, 85 (2013).
[17] L. J. Moniz, E. G. Cooch, S. P. Ellner, J. D. Nichols, and J. M. Nichols, Ecol. Model. 208, 145 (2007).

[18] L. Faes, G. Nollo, and A. Porta, Front. Physiol. 2, 1 (2011).

[19] J. Pahle, A. K. Green, C. J. Dixon, and U. Kummer, BMC Bioinf. 9, 139 (2008).

[20] J. T. Lizier, M. Prokopenko, and A. Y. Zomaya, Phys. Rev. E 77, 026110 (2008).

[21] M. Lungarella and O. Sporns, PLoS Comput. Biol. 2, 1301 (2006).

[22] J. Sun and E. M. Bollt, Physica D 267, 49 (2014).

[23] D. Chicharro and A. Ledberg, Phys. Rev. E 86, 041901 (2012).

[24] M. Paluš, V. Komárek, Z. Hrnčĩř, and K.Štěrbová, Phys. Rev. E 63, 046211 (2001).

[25] E. M. Bollt, Int. J. Bifurc. Chaos 22, 1250261 (2012).

[26] M. Palus and M. Vejmelka, Phys. Rev. E 75, 056211 (2007).

[27] K. Hlavackova-Schindler, M. Palus, M. Vejmelka, and J. Bhattacharya, Phys. Rep. 441, 1 (2007).

[28] I. Vlachos and D. Kugiumtzis, Phys. Rev. E 82, 016207 (2010).

[29] A. Kraskov, H. Stogbauer, and P. Grassberger, Phys. Rev. E 69, 066138 (2004).

[30] D. Kugiumtzis, Phys. Rev. E 87, 062918 (2013).

[31] T. M. Cover and J. A. Thomas, Elements of Information Theory, 2nd ed. (Wiley, New York, 2006).

[32] P. F. Verdes, Phys. Rev. E 72, 026222 (2005).

[33] V. A. Vakorin, O. A. Krakovska, and A. R. McIntosh, J. Neurosci. Methods 184, 152 (2009). 
[34] L. Barnett, A. B. Barrett, and A. K. Seth, Phys. Rev. Lett. 103, 238701 (2009).

[35] A. B. Barrett, L. Barnett, and A. K. Seth, Phys. Rev. E 81, 041907 (2010).

[36] D. A. Smirnov, Phys. Rev. E 87, 042917 (2013).

[37] J. T. Lizier and M. Prokopenko, Eur. Phys. J. B 73, 605 (2010).

[38] M. Wibral, R. Vicente, and M. Lindner, in Directed Information Measures in Neuroscience, edited byR. Vicente, M. Wibral, and J. T. Lizier (Springer, Berlin, 2014).

[39] F. Takens, in Dynamical Systems and Turbulence, edited by D. Rand and S. L. Young (Springer, Berlin, 1981), Vol. 898.

[40] L. Faes, G. Nollo, and A. Porta, Phys. Rev. E 83, 051112 (2011).

[41] L. Faes, D. Marinazzo, A. Montalto, and G. Nollo, IEEE Trans. Biomed. Eng. 61, 2556 (2014).

[42] L. Faes, A. Porta, and G. Nollo, Phys. Rev. E 78, 026201 (2008).

[43] G. H. Yu and C. C. Huang, Stoch. Env. Res. Risk A. 15, 462 (2001).

[44] L. Faes, A. Porta, R. Cucino, S. Cerutti, R. Antolini, and G. Nollo, Biol. Cybern. 90, 390 (2004).

[45] V. Magagnin, T. Bassani, V. Bari, M. Turiel, R. Maestri, G. D. Pinna, and A. Porta, Physiol. Meas. 32, 1775 (2011).

[46] A. Porta, T. Gnecchi-Ruscone, E. Tobaldini, S. Guzzetti, R. Furlan, and N. Montano, J. Appl. Physiol. 103, 1143 (2007).

[47] L. Faes, A. Montalto, G. Nollo, and D. Marinazzo, Comput. Cardiol. 40, 113 (2013).

[48] A. Porta, T. Bassani, V. Bari, G. D. Pinna, R. Maestri, and S. Guzzetti, IEEE Trans. Biomed. Eng. 59, 832 (2012).

[49] G. G. Berntson, J. T. Cacioppo, and K. S. Quigley, Psychophysiology 30, 183 (1993).
[50] G. Nollo, L. Faes, A. Porta, R. Antolini, and F. Ravelli, Am. J. Physiol. Heart Circ. Physiol. 288, H1777 (2005).

[51] G. Nollo, L. Faes, R. Antolini, and A. Porta, Philos. Trans. R. Soc. London Ser. A 367, 1423 (2009).

[52] L. Faes, G. Nollo, and A. Porta, J. Appl. Physiol. 114, 1657 (2013).

[53] F. Jurysta, P. van de Borne, P. F. Migeotte, M. Dumont, J. P. Lanquart, J. P. Degaute, and P. Linkowski, Clin. Neurophysiol. 114, 2146 (2003).

[54] G. Brandenberger, J. Ehrhart, F. Piquard, and C. Simon, Clin. Neurophysiol. 112, 992 (2001).

[55] C. C. H. Yang, C. W. Lai, H. Y. Lai, and T. B. J. Kuo, Neurosci. Lett. 329, 213 (2002).

[56] M. Dumont, F. Jurysta, J. P. Lanquart, P. F. Migeotte, P. van de Borne, and P. Linkowski, Clin. Neurophysiol. 115, 2031 (2004).

[57] L. Faes, G. Nollo, F. Jurysta, and D. Marinazzo, New J. Phys. 16, 105005 (2014).

[58] D. Marinazzo, M. Pellicoro, and S. Stramaglia, Comput. Math. Methods Med. 2012, 303601 (2012).

[59] A. Papana, C. Kyrtsou, D. Kugiumtzis, and C. Diks, Entropy 15, 2635 (2013).

[60] L. Faes and A. Porta, in Directed Information Measures in Neuroscience, edited by R. Vicente, M. Wibral, and J. T. Lizier (Springer, Berlin, 2014).

[61] P. O. Amblard and O. J. Michel, Entropy 15, 113 (2013).

[62] J. Geweke, J. Am. Stat. Assoc. 77, 304 (1982).

[63] L. Barnett and A. K. Seth, J. Neurosci. Methods 223, 50 (2014).

[64] S. Stramaglia, G. R. Wu, M. Pellicoro, and D. Marinazzo, Phys. Rev. E 86, 066211 (2012). 